\title{
Pseudo-Markovian viscosity solutions of fully nonlinear degenerate PPDEs
}

\author{
Ibrahim Ekren · Jianfeng Zhang
}

Received: 7 April 2016/ Accepted: 7 August 2016 / Published online: 01 December 2016 (c) The Author(s). 2016 Open Access This article is distributed under the terms of the Creative Commons Attribution 4.0 International License (http://creativecommons.org/licenses/by/4.0/), which permits unrestricted use, distribution, and reproduction in any medium, provided you give appropriate credit to the original author(s) and the source, provide a link to the Creative Commons license, and indicate if changes were made.

\begin{abstract}
In this paper, we propose a new type of viscosity solutions for fully nonlinear path-dependent PDEs. By restricting the solution to a pseudo-Markovian structure defined below, we remove the uniform non-degeneracy condition needed in our earlier works (Ekren, I, Touzi, N, Zhang, J, Ann Probab, 44:1212-1253, 2016a; Ekren, I, Touzi, N, Zhang, J, Ann Probab, 44:2507-2553, 2016b) to establish the uniqueness result. We establish the comparison principle under natural and mild conditions. Moreover, we apply our results to two important classes of PPDEs: the stochastic HJB equations and the path-dependent Isaacs equations, induced from the stochastic optimization with random coefficients and the path-dependent zero-sum game problem, respectively.
\end{abstract}

Keywords Path dependent PDEs · Viscosity solutions · Comparison principle · Stochastic HJB equations · Isaacs equations

AMS 2000 subject classifications $35 \mathrm{D} 40 \cdot 35 \mathrm{~K} 10 \cdot 60 \mathrm{H} 10 \cdot 60 \mathrm{H} 30$

\section{Introduction}

In this paper, we study the following fully nonlinear parabolic path-dependent PDE with terminal condition $u(T, \omega)=\xi(\omega)$ :

$$
\mathcal{L} u(t, \omega):=\partial_{t} u(t, \omega)+G\left(t, \omega, u, \partial_{\omega} u, \partial_{\omega \omega}^{2} u\right)=0, \quad(t, \omega) \in[0, T) \times \Omega .
$$

\footnotetext{
I. Ekren

ETH Department of Mathematics, Zürich, Switzerland

e-mail: ibrahim.ekren@math.ethz.ch.

J. Zhang $(\bowtie)$

University of Southern California, Department of Mathematics, Los Angeles, California, USA

e-mail: jianfenz@usc.edu
} 
Here $\Omega$ consists of continuous paths $\omega$ on $[0, T]$ starting from the origin, $G$ is a progressively measurable generator, and the path derivatives $\partial_{t} u, \partial_{\omega} u, \partial_{\omega \omega}^{2} u$ are defined through a functional Itô formula, initiated by Dupire (2009), see also Cont and Fournie (2013). Such an equation was first introduced by Peng (2010; 2011). In a series of papers by Ekren et al. (2014a) and Ekren et al. (2016a; 2016b), we proposed a notion of a viscosity solution for such PPDEs and established its wellposedness: existence, comparison principle, and stability. The main innovation of our notion is that, due to the lack of local compactness of the state space $\Omega$, we replace the pointwise maximum in standard PDE literature with an optimal stopping problem under certain nonlinear expectation.

Roughly speaking, the strategy in (Ekren et al. 2016a, 2016b) is a combination of partial comparison, which is a comparison between a classical semisolution and a viscosity semisolution, and a variation of the Perron's approach. In particular, when the PPDE has a classical solution, it is unique in the viscosity sense, as a direct consequence of the partial comparison. By utilizing certain path-frozen PDE (not PPDE!), in (Ekren et al. 2016b) we established the comparison in the case that the viscosity solution can be approximated by piecewise classical semisolutions taking the form:

$$
\sum_{n=0}^{\infty} v_{n}\left(\left(\mathrm{H}_{1}, \omega_{\mathrm{H}_{1}}\right), \cdots,\left(\mathrm{H}_{n}, \omega_{\mathrm{H}_{n}}\right) ; t, \omega_{t}\right) \mathbf{1}_{\left\{\mathrm{H}_{n} \leq t<\mathrm{H}_{n+1} \text { or } \mathrm{H}_{n}<\mathrm{H}_{n+1}=T=t\right\}},
$$

where $\mathrm{H}_{n}$ is an increasing sequence of stopping times with $\mathrm{H}_{0}=0$, and the mapping $(t, x) \mapsto v_{n}\left(\left(t_{1}, x_{1}\right), \cdots,\left(t_{n}, x_{n}\right) ; t, x\right)$ is in $C^{1,2}$. However, in order to obtain such smooth $v_{n}$, we need classical solutions of certain PDEs taking the form:

$$
\partial_{t} v+G_{n}\left(t, v, \partial_{x} v, \partial_{x x}^{2} v\right)=0, \quad(t, x) \in Q_{n} \subset[0, T] \times \mathbb{R}^{d} .
$$

For this purpose, in (Ekren et al. 2016b) we have to assume $G$ is uniformly nondegenerate.

The goal of this paper to is remove this uniform nondegeneracy. We note that degenerate PPDEs appear naturally in many applications, and we will present two examples in this paper. The first one is the stochastic Hamiltonian-Jacobi-Bellman equation, introduced by Peng (1992) to characterize the value function $u(t, x, \omega)$ for optimization problems with random coefficients. Peng (1992) solved the problem when there is only drift control. The general case with volatility control has been an open problem, see Peng (1999). We may view the stochastic HJB equation as a PPDE by considering $x$ as a path. This PPDE is by nature always degenerate. We shall characterize the value function as the unique viscosity solution to this degenerate PPDE. We note that in the recent work Qiu (2016) viewed the stochastic HJB equation as a backward SPDE and proved its wellposedness in the sense of Sobolev solutions. The second example is the path-dependent Isaacs equations, induced from the path-dependent zero sum game as in Pham and Zhang (2014). In order to obtain the smooth $v_{n}$ in (2), (Pham and Zhang 2014) assumes $G$ is uniformly nondegenerate and the dimension $d \leq 2$. Besides the degeneracy, our work here also allows for higher dimensions. 
We still follow the strategy in (Ekren et al. 2016b), but rely on the viscosity solution theory of PDEs, instead of the classical solution theory of PDEs as in (Ekren et al. 2016b). Namely, we will construct those $v_{n}$ via continuous (not $C^{1,2}$ !) viscosity solutions of certain path-frozen PDEs (3). However, we will establish the uniqueness of viscosity solutions in a smaller class. Notice that there is a tradeoff between the regularity of the solution and the solution class for the uniqueness. If we can establish higher regularity for the solutions (or approximate solutions), then we can prove the uniqueness within a larger solution class. In our degenerate situation, we are not able to obtain smooth $v_{n}$ in (2), but in $C^{0}$ only. As a consequence, we will establish the uniqueness only in this class, namely there is only one viscosity solution which can be approximated by piecewise Markovian viscosity solutions in the form of (2). This (piecewise) Markovian structure allows us to use the comparison principle of PDE, rather than the partial comparison of PPDE.

There is another major difficulty in the degenerate case. Note that the path frozen PDE (3) is a local PDE, with the domain $Q_{n}$ induced from the stopping times $\mathrm{H}_{n}$. However, in the degenerate case, the $\mathrm{H}_{n}$ used in (Ekren et al. 2016a, 2016b) has very bad regularity, and consequently the PDE (3) in $Q_{n}$ typically does not have a continuous viscosity solution. Strongly motivated by the recent work Bayraktar and Yao (2016), we shall use some slightly modified stopping times $\mathrm{H}_{n}$ which enjoy all the desired properties.

We remark that the present strategy, as in (Ekren et al. 2016b), relies heavily on the path frozen PDEs and the related PDE results. In particular, it uses indirectly the very deep regularity results for parabolic PDEs. In the (possibly degenerate) semilinear case, Ren et al. (2016a) and Ren (2016) studied the regularity for PPDEs directly. The more recent paper Ren et al. (2016b) established the comparison for fully nonlinear degenerate PPDEs, by introducing a regularization operator which can be viewed as the counterpart of the sup-convolution in the PDE literature. Roughly speaking, the strategy in (Ekren et al. 2016b) and the present paper is to approximate the PPDE by certain PDEs and use the solution of the latter to approximate the solution of the original PPDE. While the strategy in (Ren et al. 2016a; 2016b) is to approximate the solution of the PPDE directly and show that these approximations are solutions of certain PDEs which are close to the original PPDE in certain sense. The comparison principle in (Ren et al. 2016b), however, is also in a smaller solution class by requiring a somewhat stronger regularity on the solutions, and consequently, the coefficients of the PPDE should also have the same stronger regularity. So there is a tradeoff between (Ren et al. 2016b) and the present paper: (Ren et al. 2016b) requires stronger regularity while this paper requires certain piecewise Markovian structure. It will be indeed desirable if one could combine the two techniques and obtain the complete results, which will be left for future research.

Finally, while we focus on viscosity solutions for PPDEs, there have been different notions of solutions in the literature. First, with the smoothness in terms of Dupire's path derivatives, classical solutions were obtained by Dupire (2009) for linear PPDEs (which he called functional PDEs) and by Peng and Wang (2016) for semilinear PPDEs. Cont and Fournie (2013) extended the path derivatives to 
weaker ones which immediately provides weak solution (in the spirit of Sobolev solution) for linear PPDEs. Peng and Song (2015) studied Sobolev solutions for path-dependent HJB equations. Moreover, Cosso and Russo (2016) introduced the so called strong-viscosity solution for semilinear PPDEs as the limit of approximating classical solutions. While all the notions are consistent with classical solutions when the solutions are smooth, we emphasize that in the path-dependent case even the heat equation may not have a classical solution. Our notion of viscosity solution is a local property, thus the viscosity property can be easily verified in applications. Of course, the challenge lies in the comparison principle, which is the main focus of this paper as well as our earlier works. The Sobolev solution of (Peng and Song 2015) is a global solution and involves norm estimates, thus it is easier for uniqueness but more difficult for existence. Indeed, for the path-dependent Isaacs equations which is a typical example in our approach, it is still not clear what is the appropriate norm under which one may obtain Sobolev solution. See Pham and Zhang (2013) for a study in this direction. The strong-viscosity solution of (Cosso and Russo 2016) involves a combination of local and global properties, and is easier for uniqueness but more difficult for existence. Roughly speaking, it transforms the difficulty in our uniqueness to their existence. To the best of our knowledge, the existence of a strong-viscosity solution of (Cosso and Russo 2016) is not proven in the fully nonlinear case.

The rest of the paper is organized as follows. In "Preliminaries" Section we review the basic materials concerning PPDEs. In "Pseudo-Markovian viscosity solutions" Section we introduce pseudo Markovian viscosity solutions, and in particular the new hitting times inspired by (Bayraktar and Yao 2016). The comparison principle is proved in "Comparison principle" section, and "Existence" section is devoted to existence. In "Stochastic HJB equations" sections and "Path dependent Isaacs equation" we present two applications: the stochastic HJB equations induced from the optimization problem with random coefficients and the path-dependent Bellman-Isaacs equations induced from the zero sum stochastic differential games. Finally, some technical proofs are in the Appendix.

\section{Preliminaries}

In this section, we recall the setup in (Ekren et al. 2016b) and explain why the nondegeneracy requirement is crucial in the uniqueness proof there.

\section{The canonical setting}

Let $\Omega:=\left\{\omega \in C\left([0, T], \mathbb{R}^{d}\right): \omega_{0}=\mathbf{0}\right\}$, the set of continuous paths starting from the origin, $B$ the canonical process, $\mathbb{F}=\left\{\mathcal{F}_{t}\right\}_{0 \leq t \leq T}$ the natural filtration generated by $B, \mathbb{P}_{0}$ the Wiener measure, $\mathcal{T}$ the set of $\mathbb{F}$-stopping times, and $\Lambda:=[0, T] \times \Omega$. Here and in the sequel, for notational simplicity, we use $\mathbf{0}$ to denote vectors, matrices, or paths with appropriate dimensions whose components are all equal to 0. Moreover, let $\mathbb{S}^{d}$ denote the set of $d \times d$ symmetric matrices, and 
$x \cdot x^{\prime}:=\sum_{i=1}^{d} x_{i} x_{i}^{\prime}$ for any $x, x^{\prime} \in \mathbb{R}^{d}, \gamma: \gamma^{\prime}:=\operatorname{tr}\left[\gamma \gamma^{\prime}\right]$ for any $\gamma, \gamma^{\prime} \in \mathbb{S}^{d}$.

We say a probability measure $\mathbb{P}$ on $\mathcal{F}_{T}$ is a semimartinagle measure if $B$ is a $\mathbb{P}$ semimartingale. For every constant $L>0$, we denote by $\mathcal{P}_{L}$ the collection of all semimartingale measures $\mathbb{P}$ whose drift and diffusion characteristics are bounded by $L$ and $\sqrt{2 L}$, respectively. Denote $\mathcal{P}_{\infty}:=\cup_{L>0} \mathcal{P}_{L}$.

We next discuss regularity of random variables and processes. First, define a seminorm on $\Omega$ and a pseudometric on $\Lambda$ as follows: for any $(t, \omega),\left(t^{\prime}, \omega^{\prime}\right) \in \Lambda$,

$$
\|\omega\|_{t}:=\sup _{0 \leq s \leq t}\left|\omega_{s}\right|, \quad \mathbf{d}_{\infty}\left((t, \omega),\left(t^{\prime}, \omega^{\prime}\right)\right):=\left|t-t^{\prime}\right|^{\frac{1}{2}}+\left\|\omega_{. \wedge t}-\omega_{. \wedge t^{\prime}}^{\prime}\right\|_{T} .
$$

For a generic Euclidian space $E$, let $\mathbb{L}^{0}(\Omega ; E)$ denote the set of $\mathcal{F}_{T}$-measurable random variables $\xi, C^{0}(\Omega ; E)$ (resp. $\left.U C(\Omega ; E)\right)$ the subset of those $\xi$ continuous (resp. uniformly continuous) under $\|\cdot\|_{T}$. Similarly, let $\mathbb{L}^{0}(\Lambda ; E)$ be the set of $\mathbb{F}$ progressively measurable processes $u, C^{0}(\Lambda ; E)$ (resp. $\left.U C(\Lambda ; E)\right)$ the subset of processes continuous (resp. uniformly continuous) in $(t, \omega)$ under $\mathbf{d}_{\infty}$. We use the subscript $_{b}$ to indicate the subset of bounded elements; and we omit the notation $E$ in the spaces when $E=\mathbb{R}$. For classical solutions of PPDEs, we need further regularity of the processes. The following definition through the functional Itó's formula is due to (Ekren et al. 2016a) and is inspired by (Dupire 2009).

Definition 2.1 We say $u \in C^{1,2}(\Lambda)$ if $u \in C^{0}(\Lambda)$ and there exist $\partial_{t} u \in C^{0}(\Lambda)$, $\partial_{\omega} u \in C^{0}\left(\Lambda, \mathbb{R}^{d}\right)$, $\partial_{\omega \omega}^{2} u \in C^{0}\left(\Lambda, \mathbb{S}^{d}\right)$ such that, for any $\mathbb{P} \in \mathcal{P}_{\infty}, u$ is a $\mathbb{P}$-semimartingale satisfying:

$$
d u=\partial_{t} u d t+\partial_{\omega} u \cdot d B_{t}+\frac{1}{2} \partial_{\omega \omega}^{2} u: d\langle B\rangle_{t}, \quad 0 \leq t \leq T, \quad \mathbb{P}-a . s .
$$

We remark that the path derivatives $\partial_{t} u, \partial_{\omega} u$ and $\partial_{\omega \omega}^{2} u$, if they exist, are unique.

We finally introduce the shifted spaces. Let $0 \leq s \leq t \leq T$.

- Let $\Omega^{t}:=\left\{\omega \in C\left([t, T], \mathbb{R}^{d}\right): \omega_{t}=\mathbf{0}\right\}$ be the shifted canonical space and define $B^{t}, \mathbb{F}^{t}, \mathbb{P}_{0}^{t}, \Lambda^{t}, \mathcal{T}^{t}, \mathcal{P}_{L}^{t}, \mathcal{P}_{\infty}^{t}$ etc. in an obvious sense. In particular, $\Lambda^{t}:=$ $[t, T] \times \Omega^{t}$. Define $\|\cdot\|_{s}^{t}$ on $\Omega^{t}$ and $\mathbf{d}_{\infty}^{t}$ on $\Lambda^{t}$ in the spirit of (4), and the sets $\mathbb{L}^{0}\left(\Lambda^{t} ; E\right)$ etc. in an obvious way.

- For $\omega \in \Omega^{s}$ and $\omega^{\prime} \in \Omega^{t}$, define the concatenation path $\omega \otimes_{t} \omega^{\prime} \in \Omega^{s}$ by:

$$
\left(\omega \otimes_{t} \omega^{\prime}\right)(r):=\omega_{r} \mathbf{1}_{[s, t)}(r)+\left(\omega_{t}+\omega_{r}^{\prime}\right) \mathbf{1}_{[t, T]}(r), \text { for all } r \in[s, T] .
$$

- Let $\xi \in \mathbb{L}^{0}\left(\Omega^{s}\right)$ and $X \in \mathbb{L}^{0}\left(\Lambda^{s}\right)$. For $(t, \omega) \in \Lambda^{s}$, define $\xi^{t, \omega} \in \mathbb{L}^{0}\left(\Omega^{t}\right)$ and $X^{t, \omega} \in \mathbb{L}^{0}\left(\Lambda^{t}\right)$ by:

$$
\xi^{t, \omega}\left(\omega^{\prime}\right):=\xi\left(\omega \otimes_{t} \omega^{\prime}\right), \quad X^{t, \omega}\left(\omega^{\prime}\right):=X\left(\omega \otimes_{t} \omega^{\prime}\right), \text { for all } \omega^{\prime} \in \Omega^{t} .
$$

It is clear that, for any $(t, \omega) \in \Lambda$ and any $u \in C^{0}(\Lambda)$, we have $u^{t, \omega} \in C^{0}\left(\Lambda^{t}\right)$. The spaces introduced above enjoy the same property. 


\section{Viscosity solution of PPDEs}

Our PPDE takes the form of (1) with a terminal condition $u(T, \omega)=\xi(\omega)$. We say $u \in C^{1,2}(\Lambda)$ is a classical solution (resp. supersolution, subsolution) of PPDE (1) if

$$
\mathcal{L} u(t, \omega)=(\text { resp. } \leq, \geq) 0, \quad \forall(t, \omega) \in[0, T) \times \Omega .
$$

The definition of viscosity solution is more involved. First, for any $\xi \in \mathbb{L}^{0}\left(\Omega^{t}\right)$ with appropriate integrability, we introduce the following nonlinear expectations:

$$
\overline{\mathcal{E}}_{t}^{L}[\xi]:=\sup _{\mathbb{P} \in \mathcal{P}_{L}^{t}} \mathbb{E}^{\mathbb{P}}[\xi] \text { and } \underline{\mathcal{E}}_{t}^{L}[\xi]:=\inf _{\mathbb{P} \in \mathcal{P}_{L}^{t}} \mathbb{E}^{\mathbb{P}}[\xi]=-\overline{\mathcal{E}}_{t}^{L}[-\xi] .
$$

Next, for any $t \in[0, T]$ and $\varepsilon>0$, we define a hitting time:

$$
\widehat{\mathrm{H}}_{\varepsilon}^{t}:=\inf \left\{s>t:\left|B_{s}^{t}\right| \geq \varepsilon\right\} \wedge(t+\varepsilon) \wedge T .
$$

Now for $u \in \mathbb{L}^{0}(\Lambda)$ with appropriate integrability, we introduce the following classes of test functions: for any $L>0$ and $(t, \omega) \in[0, T) \times \Omega$,

$$
\begin{aligned}
& \underline{\mathcal{A}}^{L} u(t, \omega):=\left\{\varphi \in C^{1,2}\left(\Lambda^{t}\right):\left(\varphi-u^{t, \omega}\right)_{t}=0=\inf _{\tau \in \mathcal{T}^{t}} \mathcal{E}_{t}^{L}\left[\left(\varphi-u^{t, \omega}\right)_{\left.\left.\tau \wedge \widehat{\mathrm{H}}_{\varepsilon}^{t}\right] \text { for some } \varepsilon>0\right\},}\right.\right. \\
& \overline{\mathcal{A}}^{L} u(t, \omega):=\left\{\varphi \in C^{1,2}\left(\Lambda^{t}\right):\left(\varphi-u^{t, \omega}\right)_{t}=0=\sup _{\tau \in \mathcal{T}^{t}} \overline{\mathcal{E}}_{t}^{L}\left[\left(\varphi-u^{t, \omega}\right)_{\left.\left.\tau \wedge \widehat{\mathrm{H}}_{\varepsilon}^{t}\right] \text { for some } \varepsilon>0\right\} .}\right.\right.
\end{aligned}
$$

Definition 2.2 Let $u \in \mathbb{L}^{0}(\Lambda)$ with appropriate integrability and $L>0$. We say $u$ is a $\mathcal{P}_{L}$-viscosity subsolution (resp. supersolution) of PPDE (1) if, for any $(t, \omega) \in[0, T) \times \Omega$ and any $\varphi \in \underline{\mathcal{A}}^{L} u(t, \omega)\left(\right.$ resp. $\left.\varphi \in \overline{\mathcal{A}}^{L} u(t, \omega)\right)$ :

$$
\mathcal{L}^{t, \omega} \varphi(t, \boldsymbol{0}):=\partial_{t} \varphi(t, \boldsymbol{0})+G^{t, \omega}\left(\cdot, \varphi, \partial_{\omega} \varphi, \partial_{\omega \omega}^{2} \varphi\right)(t, \boldsymbol{0}) \geq \quad(\text { resp. } \leq) 0 .
$$

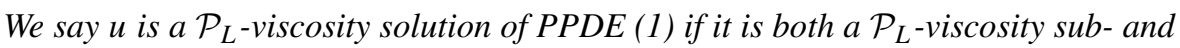
supersolution.

We remark that to establish the viscosity theory certain semi-regularity is required for semisolutions, as introduced in (Ekren et al. 2016a, 2016b). Moreover, the smooth test processes $\varphi$ in 8 can actually be restricted to parabolas, and thus the definition can be rewritten in terms of semi-jets, see (Ren et al. 2014).

\section{Viscosity solution of PDEs}

In this subsection, we consider the following PDE on an open domain $Q \subset[0, T) \times$ $\mathbb{R}^{d}$ :

$$
\mathbb{L} v(t, x):=\partial_{t} v(t, x)+g\left(t, x, v, \partial_{x} v, \partial_{x x} v\right)=0, \quad(t, x) \in Q .
$$

We shall introduce two notions of viscosity solutions, one is adapted from Definition 2.2, and the other is the standard one in PDE literature, see e.g. (Crandall et al. 1992) and (Fleming and Soner 2006).

Definition 2.3 Let $v: Q \rightarrow \mathbb{R}$ be measurable with certain integrability. 
(i) For some $L>0$, we say $v$ is a $\mathcal{P}_{L}$-viscosity subsolution of PDE (9) if, for any $(t, x) \in Q$,

$$
\begin{aligned}
\mathbb{L} \varphi(t, x) & \geq 0, \quad \forall \varphi \in \underline{\mathcal{A}}^{L} v(t, x), \text { where } \\
\underline{\mathcal{A}}^{L} v(t, x) & :=\left\{\varphi \in C^{1,2}(Q): \exists \varepsilon>0 \text { s.t. }[\varphi-v](t, x)=0\right. \\
& \left.=\inf _{\tau \in \mathcal{T}^{t}: \tau \leq \widehat{\mathrm{H}}_{\varepsilon}^{t}} \underline{\mathcal{E}}_{t}^{L}\left[[\varphi-v]\left(\tau, x+B_{\tau}^{t}\right)\right]\right\} .
\end{aligned}
$$

(ii) We say $v$ is a Crandall-Lions viscosity subsolution of PDE (9) if, for any $(t, x) \in Q$,

$\mathbb{L} \varphi(t, x) \geq 0, \quad \forall \varphi \in \underline{\mathcal{A}} v(t, x)$, where

$\underline{\mathcal{A}} v(t, x):=\left\{\varphi \in C^{1,2}(Q): \exists \varepsilon>0\right.$ s.t. $\left.[\varphi-v](t, x)=0=\inf _{(s, y) \in Q:|s-t|+|y-x| \leq \varepsilon}[\varphi-v](s, y)\right\}$.

(iii) We define the corresponding viscosity supersolution and the viscosity solution in an obvious way.

Remark 2.4 (i) When $\varepsilon>0$ is small enough, we have $\left(s, x+B_{s}^{t}\right) \in Q$ for all $s \leq \widehat{\mathrm{H}}_{\varepsilon}^{t}$. Thus the $\underline{\mathcal{A}}^{L} v(t, x)$ in (10) is well defined.

(ii) It is clear that $\underline{\mathcal{A}} v(t, x) \subset \underline{\mathcal{A}}^{L} v(t, x)$. Then a $\mathcal{P}_{L}$-viscosity subsolution is a Crandall-Lions viscosity subsolution. In general, these notions might not be equivalent.

(iii) Due to (ii), formally it could be easier to prove the comparison principle for $\mathcal{P}_{L}$-viscosity semisolutions than for Crandall-Lions viscosity semisolutions. It will be interesting to explore such a possibility.

\section{The degeneracy of $G$}

In this subsection, we explain why the non-degeneracy requirement is crucial for the comparison principle in (Ekren et al. 2016b), and how we overcome the difficulties in this paper.

A key element in the strategy of (Ekren et al. 2016b) is the following path frozen $\mathrm{PDE}$ (not PPDE!): for fixed $(t, \omega) \in[0, T) \times \Omega$ and $\varepsilon>0$,

$$
\partial_{t} v+G\left(s, \omega_{\cdot \wedge t}, v, \partial_{x} v, \partial_{x x} v\right)=0, \quad(s, x) \in \widehat{Q}_{\varepsilon}^{t}:=[t,(t+\varepsilon) \wedge T) \times\left\{x \in \mathbb{R}^{d}:|x|<\varepsilon\right\}
$$

We emphasize that at above the path $\omega$ in $G$ is frozen at $t$ and thus the equation is a (deterministic) PDE. Moreover, the domain $\widehat{Q}_{\varepsilon}^{t}$ is induced by the hitting time $\widehat{\mathrm{H}}_{\varepsilon}^{t}$, indeed, we have $\left(s, B_{s}^{t}\right) \in \widehat{Q}_{\varepsilon}^{t}$ for $s<\widehat{\mathrm{H}}_{\varepsilon}^{t}$.

In order to construct smooth test functions, we let $G_{\varepsilon}$ be a smooth mollifier of $G$ and require the following mollified path frozen PDE (with smooth boundary condition) has a classical solution:

$$
\partial_{t} v+G_{\varepsilon}\left(s, \omega_{\cdot \wedge t}, v, \partial_{x} v, \partial_{x x} v\right)=0, \quad(s, x) \in \widehat{Q}_{\varepsilon}^{t}
$$


In the PDE literature, one typically needs uniform non-degeneracy of $G_{\varepsilon}$ in terms of $\gamma$, namely there exists a constant $c_{0}>0$ such that

$$
G_{\varepsilon}\left(\cdot, \gamma+\gamma^{\prime}\right)-G_{\varepsilon}(\cdot, \gamma) \geq c_{0} \operatorname{tr}\left(\gamma^{\prime}\right), \quad \forall \gamma, \gamma^{\prime} \in \mathbb{S}^{d} \text { with } \gamma^{\prime} \geq \mathbf{0} .
$$

Moreover, for Bellman-Isaacs equations, one may obtain classical solution only when $d \leq 2$, even if $G_{\varepsilon}$ is uniformly non-degenerate.

We note that the classical solution of (13) is used to prove the partial comparison principle, namely the comparison between a classical semisolution and a viscosity semisolution. Our first observation is that, since we are utilizing PDE results, we can use the comparison principle for viscosity solutions of PDE directly. We note that by doing this we are using the regularities of PDEs indirectly, because the comparison principle in PDE literature relies on the regularities through a regularization procedure. Nevertheless, this allows us to use the viscosity theory rather than classical solutions of PDEs. However, this requires our viscosity semisolutions to have certain piecewise Markovian structure, which we will call pseudo-Markovian, thus our comparison principle will be within a smaller class than that in (Ekren et al. 2016b).

There is another difficulty in the degenerate case, even for the viscosity theory of PDEs. Notice that the PDEs (12) and (13) are on a bounded domain $\widehat{Q}_{\varepsilon}^{t}$, not on the whole space. As we see in the following example, in the degenerate case such a PDE with a smooth boundary condition may not have a continuous viscosity solution.

Example 2.5 Consider the following degenerate PDE:

$$
\begin{aligned}
\partial_{t} v & =0,(t, x) \in \widehat{Q}_{\varepsilon}^{0} ; \quad v(t, x)=t,(t, x) \in \partial \widehat{Q}_{\varepsilon}^{0} \\
& :=\{(t, x): t=\varepsilon,|x| \leq \varepsilon \text { or } t<\varepsilon,|x|=\varepsilon\} .
\end{aligned}
$$

Then clearly the candidate solution should be: $v(t, x)=\varepsilon \mathbf{1}_{\widehat{Q}_{\varepsilon}^{0}}(t, x)+t \mathbf{1}_{\partial \widehat{Q}_{\varepsilon}^{0}}(t, x)$, which, unfortunately, is discontinuous on $\{(t, x): t<\varepsilon,|x|=\varepsilon\}$.

Inspired by (Bayraktar and Yao 2016), we overcome this difficulty by modifying the hitting times. While we will study the new hitting time in details in next section, we present a special case here to see how it helps overcome the difficulty in the example above. Consider the following hitting time:

$$
\mathrm{H}_{\varepsilon}:=\inf \left\{t \geq 0: t+\left|B_{t}\right| \geq \varepsilon\right\},
$$

which would induce a domain, changing from a cylinder to a cone:

$$
\begin{aligned}
Q_{\varepsilon} & :=\left\{(t, x) \in[0, \varepsilon) \times \mathbb{R}^{d}: t+|x|<\varepsilon\right\}, \\
\partial Q_{\varepsilon} & :=\left\{(t, x) \in[0, \varepsilon) \times \mathbb{R}^{d}: t+|x|=\varepsilon\right\} .
\end{aligned}
$$

Example 2.6 Consider the following degenerate PDE:

$$
\partial_{t} v=0, \quad(t, x) \in Q_{\varepsilon} ; \quad v(t, x)=t,(t, x) \in \partial Q_{\varepsilon} .
$$

Then the solution is: $v(t, x)=\varepsilon-|x|$, which is continuous on the whole domain $Q_{\varepsilon} \cup \partial Q_{\varepsilon}$. 


\section{Pseudo-Markovian viscosity solutions}

Our PPDE of interest is (1) with a terminal condition $u(T, \omega)=\xi(\omega)$. We shall assume the following standing assumptions.

Assumption 3.1 (i) The PPDE is parabolic, namely $G$ is non-decreasing in $\gamma$; (ii) $G$ is uniformly Lipschitz continuous in $(y, z, \gamma)$ with Lipschitz constant L;

(iii) $G$ is continuous in $(t, \omega), G(\cdot, 0, \boldsymbol{0}, \boldsymbol{0})$ is bounded, and $\xi \in C_{b}^{0}(\Omega)$.

Throughout the paper, for notational simplicity we denote: for any process $\varphi$ and $s<t$

$$
L_{1}:=L+1, \quad \varphi_{s, t}:=\varphi_{t}-\varphi_{s} .
$$

\section{Hitting times}

As explained in Subsection "The degeneracy of $\boldsymbol{G}$ ", we shall introduce a new type of hitting time, strongly motivated by the recent work (Bayraktar and Yao 2016). Given $R>0, t \in[0, T)$ and $x \in \mathbb{R}^{d}$ with $|x| \leq R$, define

$$
\mathrm{H}^{t, x, R}\left(B_{.}^{t}\right):=\inf \left\{s \geq t:\left|x+B_{s}^{t}\right|+L_{1}(s-t) \geq R\right\} \wedge T .
$$

This hitting time enjoys certain useful properties.

Lemma 3.2 For any $(t, x, R), \tau \in \mathcal{T}^{t}$ with $\tau \leq \mathrm{H}^{t, x, R}$, and $\delta>0$, we have

$$
\begin{aligned}
& \mathrm{H}^{t, x, R}\left(B_{.}^{t}\right)=\mathrm{H}^{\tau, x+B_{\tau}^{t}, R-L(\tau-t)}\left(B^{t}-B_{\tau}^{t}\right), \\
& \sup _{\mathbb{P} \in \mathcal{P}_{L}^{t}} \mathbb{P}\left(\mathrm{H}^{t, 0, R}<(t+\delta) \wedge T\right) \leq C_{R} \delta .
\end{aligned}
$$

Moreover, $\mathrm{H}^{t, x, R}$ is increasing in $R$, and has the following regularities:

$$
\begin{gathered}
\overline{\mathcal{E}}_{t}^{L}\left[\left|\mathrm{H}^{t, x_{1}, R_{1}}-\mathrm{H}^{t, x_{2}, R_{2}}\right|\right] \leq\left|x_{1}-x_{2}\right|+\left|R_{1}-R_{2}\right|, \\
\left|x_{1}\right| \leq R_{1},\left|x_{2}\right| \leq R_{2} ; \\
\overline{\mathcal{E}}_{t}^{L}\left[\left|\mathrm{H}^{t, x, R}\left(B^{t}\right)-\mathrm{H}^{\tau, x, R}\left(B^{t}-B_{\tau}^{t}\right)\right|\right] \leq C \overline{\mathcal{E}}_{t}^{L}[\sqrt{\tau-t}], \\
0 \leq t \leq \tau \leq \mathrm{H}^{t, x, R},|x| \leq R .
\end{gathered}
$$

Proof First, (19) and the monotonicity of $\mathrm{H}^{t, x}, R$ in $R$ are obvious. Next, for any $\delta>0$, if $L_{1} \delta \geq \frac{R}{2}$, then (20) becomes trivial. Now assume $L_{1} \delta \leq \frac{R}{2}$. For any $\mathbb{P} \in \mathcal{P}_{L}^{t}$,

$$
\begin{aligned}
\mathbb{P}\left(\mathrm{H}^{t, 0, R}<(t+\delta) \wedge T\right) & \leq \mathbb{P}\left(\sup _{t \leq s \leq t+\delta}\left|B_{s}^{t}\right|+L_{1} \delta \geq R\right) \leq \mathbb{P}\left(\sup _{t \leq s \leq t+\delta}\left|B_{s}^{t}\right| \geq \frac{R}{2}\right) \\
& \leq \frac{4}{R^{2}} \mathbb{E}^{\mathbb{P}}\left[\sup _{t \leq s \leq t+\delta}\left|B_{s}^{t}\right|^{2}\right] \leq C_{R} \delta .
\end{aligned}
$$


By the arbitrariness of $\mathbb{P}$, this implies (20). Moreover, (22) follows directly from (19), (21), and the following simple estimate: $\overline{\mathcal{E}_{t}^{L}}\left[\left|B_{\tau}^{t}\right|\right] \leq C \overline{\mathcal{E}_{t}^{L}}[\sqrt{\tau-t}]$.

To prove (21), we assume without loss of generality that $t=0$ and denote $\tau_{i}:=$ $\mathrm{H}^{0, x_{i}, R_{i}}, i=1,2$, and $\Delta \varphi:=\varphi_{2}-\varphi_{1}$ for $\varphi=x, R, \tau$. On $\left\{\tau_{1}<\tau_{2}\right\} \in \mathcal{F}_{\tau_{1}}$ and under each $\mathbb{P} \in \mathcal{P}_{L}$, we have

$$
\begin{gathered}
\left|x_{1}+B_{\tau_{1}}\right|+L_{1} \tau_{1}=R_{1}, \quad\left|x_{2}+B_{\tau_{2}}\right|+L_{1} \tau_{2} \leq R_{2} \\
\Rightarrow \Delta R \geq \mathbb{E}_{\tau_{1}}^{\mathbb{P}}\left[\left|x_{2}+B_{\tau_{2}}\right|+L_{1} \tau_{2}\right]-\left[\left|x_{1}+B_{\tau_{1}}\right|+L_{1} \tau_{1}\right] \\
\geq\left|x_{2}+\mathbb{E}_{\tau_{1}}^{\mathbb{P}}\left[B_{\tau_{2}}\right]\right|-\left|x_{1}+B_{\tau_{1}}\right|+L_{1} \mathbb{E}_{\tau_{1}}^{\mathbb{P}}[\Delta \tau] \\
\quad \geq L_{1} \mathbb{E}_{\tau_{1}}^{\mathbb{P}}[\Delta \tau]-|\Delta x|-\left|\mathbb{E}_{\tau_{1}}^{\mathbb{P}}\left[B_{\tau_{1}, \tau_{2}}\right]\right| \\
\quad \geq L_{1} \mathbb{E}_{\tau_{1}}^{\mathbb{P}}[\Delta \tau]-|\Delta x|-L \mathbb{E}_{\tau_{1}}^{\mathbb{P}}[\Delta \tau]=\mathbb{E}_{\tau_{1}}^{\mathbb{P}}[\Delta \tau]-|\Delta x| \\
\Rightarrow \mathbb{E}_{\tau_{1}}^{\mathbb{P}}[\Delta \tau] \leq|\Delta x|+|\Delta R| .
\end{gathered}
$$

This implies that

$$
\mathbb{E}^{\mathbb{P}}\left[\left(\tau_{2}-\tau_{1}\right) \mathbf{1}_{\left\{\tau_{1}<\tau_{2}\right\}}\right] \leq[|\Delta x|+|\Delta R|] \mathbb{P}\left(\tau_{1}<\tau_{2}\right) .
$$

Similarly, we have $\mathbb{E}^{\mathbb{P}}\left[\left(\tau_{1}-\tau_{2}\right) \mathbf{1}_{\left\{\tau_{2}<\tau_{1}\right\}}\right] \leq[|\Delta x|+|\Delta R|] \mathbb{P}\left(\tau_{2}<\tau_{1}\right)$. Then $\mathbb{E}^{\mathbb{P}}[|\Delta \tau|] \leq[|\Delta x|+|\Delta R|]$, and (21) follows from the arbitrariness of $\mathbb{P} \in \mathcal{P}_{L}$.

Remark 3.3 (i) The regularities (21) and (22) are in $\mathbb{L}^{1}$-sense. The hitting time $\widehat{\mathrm{H}}_{\varepsilon}^{t}$ in (7) shares these properties in the uniformly non-degenerate case, but does not in the degenerate case. The work (Bayraktar and Yao 2016) introduced a different hitting time which has stronger regulairty:

$$
\mathrm{H}_{\varepsilon}^{*}:=\inf \left\{t \geq 0: t+\sup _{0 \leq s \leq t}\left|B_{s}\right| \geq \varepsilon\right\}
$$

One can easily show that $\mathrm{H}_{\varepsilon}^{*}$ is Lipschitz continuous in $\omega$ in the pathwise sense:

$$
\left|\mathrm{H}_{\varepsilon}^{*}(\omega)-\mathrm{H}_{\varepsilon}^{*}(\tilde{\omega})\right| \leq\|\omega-\tilde{\omega}\|_{T} .
$$

However, $\mathrm{H}_{\varepsilon}^{*}$ does not share the Markovian property in the sense of (19):

$$
\mathrm{H}_{\varepsilon}^{*} \neq \mathrm{H}_{\varepsilon}^{*, \tau, B_{\tau}} \text { for } \tau<\widehat{\mathrm{H}}_{\varepsilon}, \quad \text { where } \widehat{\mathrm{H}}_{\varepsilon}^{*, t, x}:=\inf \left\{s \geq t: s+\sup _{t \leq r \leq s}\left|x+B_{r}^{t}\right| \geq \varepsilon\right\} \text {. }
$$

In this paper, we need both the regularity and the Markovian structure, in order to utilize the viscosity theory of PDEs.

(ii) The regularities (21) and (22) are under nonlinear expectation. Under standard (linear) expectation, such regularities have been well understood, see e.g. (Mikulevicious 1987, Mikulevicius and Rozovskii 1999).

(iii) For any $\varepsilon>0$, there exist $0<\varepsilon_{1}, \varepsilon_{2}<\varepsilon$ such that

$$
\widehat{\mathrm{H}}_{\varepsilon_{1}}^{t} \leq \mathrm{H}^{t, \boldsymbol{0}, \varepsilon}, \quad \mathrm{H}^{t, \boldsymbol{0}, \varepsilon_{2}} \leq \widehat{\mathrm{H}}_{\varepsilon}^{t} .
$$

Then clearly Definition 2.2 remains equivalent if we replace the $\widehat{\mathrm{H}}_{\varepsilon}^{t}$ in (8) with $\mathrm{H}^{t, 0, \varepsilon}$. Moreover, the optimal stopping problem, which is required in (Ekren et al. 2016a, 2016b) and proved in (Ekren et al. 2014b), becomes a lot easier if we use $\mathrm{H}^{t, 0, \varepsilon}$ due to the regularities in Lemma 3.2. 
The next property will be crucial to pass the local structure to a global one. Fix $\varepsilon>0$, define

$$
\begin{aligned}
\mathrm{H}_{0}^{\varepsilon}:=0, \quad \mathrm{H}_{n+1}^{\varepsilon} & :=\mathrm{H}_{n}^{\mathrm{H}_{n}^{\varepsilon}, 0, \varepsilon}\left(B .-B_{\mathrm{H}_{n}^{\varepsilon}}\right)=\inf \left\{t \geq \mathrm{H}_{n}^{\varepsilon}:\left|B_{\mathrm{H}_{n}^{\varepsilon}, t}\right|+L_{1}\left(t-\mathrm{H}_{n}^{\varepsilon}\right)\right. \\
& \geq \varepsilon\} \wedge T, n \geq 0 .
\end{aligned}
$$

Lemma 3.4 For any $\varepsilon>0$,

$$
\bigcap_{n \geq 1}\left\{\mathrm{H}_{n}^{\varepsilon}<T\right\}=\emptyset \quad \text { and } \sup _{\mathbb{P} \in \mathcal{P}_{L}} \mathbb{P}\left[\mathrm{H}_{n}^{\varepsilon}<T\right] \leq \frac{C}{n \varepsilon^{2}} .
$$

Proof First, clearly $\mathrm{H}_{n}^{\varepsilon}$ is nondecreasing, and thus $\mathrm{H}_{\infty}^{\varepsilon}:=\lim _{n \rightarrow \infty} \mathrm{H}_{n}^{\varepsilon} \leq T$ exists. Note that,

$$
\bigcap_{n \geq 1}\left\{\mathrm{H}_{n}^{\varepsilon}<T\right\} \subset \bigcap_{n \geq 1}\left\{\left|B_{\mathrm{H}_{n}^{\varepsilon}, \mathrm{H}_{n+1}^{\varepsilon}}\right|+L_{1}\left(\mathrm{H}_{n+1}^{\varepsilon}-\mathrm{H}_{n}^{\varepsilon}\right)=\varepsilon\right\} .
$$

Since $\lim _{n \rightarrow \infty} B_{\mathrm{H}_{n}^{\varepsilon}}=B_{\mathrm{H}_{\infty}^{\varepsilon}}$, clearly the right side above is empty, then so is the left side.

Next, for any $n \geq 1$,

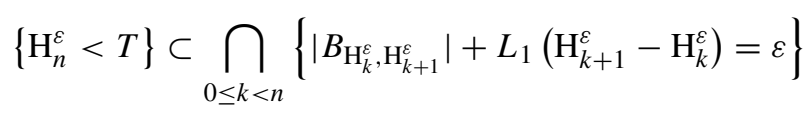

Note that

$$
\left(\left|B_{\mathrm{H}_{k}^{\varepsilon}}, \mathrm{H}_{k+1}^{\varepsilon}\right|+L_{1}\left(\mathrm{H}_{k+1}^{\varepsilon}-\mathrm{H}_{k}^{\varepsilon}\right)\right)^{2} \leq 2\left|B_{\mathrm{H}_{k}^{\varepsilon}}, \mathrm{H}_{k+1}^{\varepsilon}\right|^{2}+C\left(\mathrm{H}_{k+1}^{\varepsilon}-\mathrm{H}_{k}^{\varepsilon}\right) .
$$

Then

$$
\begin{aligned}
\left\{\mathrm{H}_{n}^{\varepsilon}<T\right\} & \subset \bigcap_{0 \leq k<n}\left\{2\left|B_{\mathrm{H}_{k}^{\varepsilon}, \mathrm{H}_{k+1}^{\varepsilon}}\right|^{2}+C\left(\mathrm{H}_{k+1}^{\varepsilon}-\mathrm{H}_{k}^{\varepsilon}\right) \geq \varepsilon^{2}\right\} \\
& \subset\left\{\sum_{k=0}^{n-1}\left[2\left|B_{\mathrm{H}_{k}^{\varepsilon}, \mathrm{H}_{k+1}^{\varepsilon}}\right|^{2}+C\left(\mathrm{H}_{k+1}^{\varepsilon}-\mathrm{H}_{k}^{\varepsilon}\right)\right] \geq n \varepsilon^{2}\right\} \\
& \subset\left\{2 \sum_{k=0}^{n-1}\left|B_{\mathrm{H}_{k}^{\varepsilon}, \mathrm{H}_{k+1}^{\varepsilon}}\right|^{2}+C \geq n \varepsilon^{2}\right\} .
\end{aligned}
$$

Now for any $\mathbb{P} \in \mathcal{P}_{L}$,

$$
\mathbb{P}\left(\mathrm{H}_{n}^{\varepsilon}<T\right) \leq \frac{1}{n \varepsilon^{2}} \mathbb{E}^{\mathbb{P}}\left[2 \sum_{k=0}^{n-1}\left|B_{\mathrm{H}_{k}^{\varepsilon}, \mathrm{H}_{k+1}^{\varepsilon}}\right|^{2}+C\right] \leq \frac{C}{n \varepsilon^{2}} .
$$

By the arbitrariness of $\mathbb{P} \in \mathcal{P}_{L}$ we obtain the second claim immediately. 


\section{Piecewise Markovian processes}

For any $t \in[0, T]$ and $\varepsilon>0$, denote

$$
\begin{aligned}
Q_{t}^{\varepsilon}:=\left\{(s, x) \in(t, T] \times \mathbb{R}^{d}:|x|+L_{1}(s-t)<\varepsilon\right\} ; \\
\partial Q_{t}^{\varepsilon}:=\left\{(s, x) \in(t, T] \times \mathbb{R}^{d}:|x|+L_{1}(s-t)=\varepsilon\right\} \\
\cup\left\{(T, x):|x|+L_{1}(T-t) \leq \varepsilon\right\} ; \\
\widehat{Q}_{t}^{\varepsilon}:=Q_{t}^{\varepsilon} \cup \partial Q_{t}^{\varepsilon} \cup\{(t, \mathbf{0})\} ; \\
\Pi_{n}^{\varepsilon}=\left\{\pi_{n}=\left(t_{i}, x_{i}\right)_{1 \leq i \leq n}: 0=t_{0}<t_{1}<\cdots<t_{n}<T,\left(t_{i}, x_{i}\right) \in \partial Q_{t_{i-1}}^{\varepsilon}, 1 \leq i \leq n\right\} ; \\
D_{n+1}^{\varepsilon}:=\left\{\left(\pi_{n} ; t, x\right): \pi_{n} \in \Pi_{n}^{\varepsilon},(t, x) \in \widehat{Q}_{t_{n}}^{\varepsilon}\right\} ; \\
\pi_{n}^{\varepsilon}(\omega):=\left(\mathrm{H}_{i}^{\varepsilon}(\omega), \omega_{\mathrm{H}_{i}^{\varepsilon}(\omega)}-\omega_{\mathrm{H}_{i-1}^{\varepsilon}(\omega)}\right)_{1 \leq i \leq n} .
\end{aligned}
$$

In light of (2), we introduce processes with the following piecewise Markovian structure.

Definition 3.5 Let $\varepsilon>0$. We say a process $u \in \mathbb{L}^{0}(\Lambda)$ is $\varepsilon$-Markovian, denoted as $u \in \mathcal{M}_{\varepsilon}(\Lambda)$, if there exist deterministic functions $v_{n}: D_{n+1}^{\varepsilon} \rightarrow \mathbb{R}, n \geq 0$, satisfying:

(i) (2) holds, namely

$$
\left.u(t, \omega)=\sum_{n=0}^{\infty} v_{n}\left(\pi_{n}^{\varepsilon}(\omega) ; t, \omega_{t}-\omega_{\mathrm{H}_{n}(\omega)}\right) \mathbf{1}_{\left\{\mathrm{H}_{n}^{\varepsilon} \leq t<\mathrm{H}_{n+1}^{\varepsilon}\right.} \text { or } \mathrm{H}_{n}^{\varepsilon}<\mathrm{H}_{n+1}^{\varepsilon}=T=t\right\}
$$

(ii) For all $\pi_{n}=\left(t_{i}, x_{i}\right)_{1 \leq i \leq n} \in \Pi_{n}^{\varepsilon}$ and $(t, x) \in \partial Q_{t_{n}}^{\varepsilon}$, the following compatibility condition holds

$$
v_{n}\left(\pi_{n} ; t, x\right)=v_{n+1}\left(\pi_{n},(t, x) ; t, 0\right) .
$$

(iii) Each $v_{n}, n \geq 0$, is continuous in $D_{n+1}^{\varepsilon}$.

Remark 3.6 $(i)$ The continuity of $v_{n}$ and compatibility (29) imply that $u$ is continuous in time.

(ii) We do not require $v_{n}\left(\pi_{n} ; \cdot\right)$ to be continuous on $\left\{\left(t_{n}, x\right): 0<|x| \leq \varepsilon\right\}$. However, for any $\delta>0$ small, $v_{n}$ is (uniformly) continuous on the following compact set

$$
D_{n+1}^{\varepsilon, \delta}:=\left\{\left(\pi_{n} ; t, x\right) \in D_{n+1}^{\varepsilon}: t_{i}-t_{i-1} \geq \delta, i=1, \cdots, n, \text { and } t-t_{n} \geq \delta\right\} .
$$

It turns out that this uniform continuity and the continuity of $v_{n}\left(\pi_{n} ; \cdot\right)$ at $\left(t_{n}, \boldsymbol{0}\right)$ is enough for our comparison result.

(iii) In (Ekren et al. 2016b) we imposed a technical condition Assumption 3.5 to ensure the constructed $v_{n}$ will be uniformly continuous in $D_{n+1}^{\varepsilon}$. This condition is not needed here because of the introduction of our new hitting time.

Moreover, we may extend all the notations to the shifted spaces: given $0 \leq t<T$, 


$$
\mathrm{H}_{n}^{t, \varepsilon}, \quad \Pi_{n}^{t, \varepsilon}, \quad \pi_{n}^{t, \varepsilon}, \quad \mathcal{M}_{\varepsilon}\left(\Lambda^{t}\right), \text { etc. }
$$

\section{Pseudo-Markovian viscosity solution}

We provide the following notion of viscosity solutions.

Definition 3.7 We say $u$ is a pseudo-Markovian $\mathcal{P}_{L^{-}}$-viscosity subsolution (resp. Crandall-Lions viscosity subsolution) of PPDE (1) at $(t, \omega) \in[0, T) \times \Omega$ if, for any $\varepsilon>0$, there exists $u^{t, \omega, \varepsilon} \in \mathcal{M}_{\varepsilon}\left(\Lambda^{t}\right)$ with corresponding $\left\{v_{n}, n \geq 1\right\}$, such that

(i) for each $\pi_{n}=\left(t_{i}, x_{i}\right)_{1 \leq i \leq n} \in \Pi_{n}^{t, \varepsilon}, v_{n}\left(\pi_{n} ; \cdot\right)$ is a $\mathcal{P}_{L}$-viscosity subsolution (resp. Crandall-Lions viscosity subsolution) to the following PDE:

$$
\begin{aligned}
\mathbb{L}^{t, \omega, \pi_{n}} v_{n}\left(\pi_{n} ; s, x\right) & :=\partial_{t} v_{n}\left(\pi_{n} ; s, x\right)+G\left(s, \omega \otimes_{t} \omega^{\pi_{n}}, v_{n}, \partial_{x} v_{n}, \partial_{x x}^{2} v_{n}\right) \\
& =0, \quad(s, x) \in Q_{t_{n}}^{\varepsilon},
\end{aligned}
$$

where $\omega^{\pi_{n}}$ is the linear interpolation of $(t, \boldsymbol{0}),\left(t_{i}, \sum_{j=1}^{i} x_{j}\right)_{1 \leq i \leq n},\left(T, \sum_{j=1}^{n} x_{j}\right)$,

(ii) $u^{t, \omega, \varepsilon} \leq u^{t, \omega}$ on $\Lambda^{t}$ and $\lim _{\varepsilon \rightarrow 0} u^{t, \omega, \varepsilon}(t, \boldsymbol{\theta})=u(t, \omega)$.

We define a pseudo Markovian viscosity supersolution similarly, and we call $u$ a pseudo-Markovian viscosity solution if it is both a pseudo Markovian viscosity subsolution and supersolution.

Remark 3.8 (i) Definition 2.2 is completely local. Definition 3.7 is in between local and global. The $u^{t, \omega, \varepsilon}$ may depend on $(t, \omega)$ and we require the convergence of $u^{t, \omega, \varepsilon}$ only at $(t, \omega)$. In this sense our definition is local. However, the viscosity property of $u^{t, \omega, \varepsilon}$ and the inequality $u^{t, \omega, \varepsilon} \leq u^{t, \omega}$ hold on $\Lambda^{t}$ and in this sense the definition is global.

(ii) In Definition 3.7 the $v_{n}$ is required to satisfy the path frozen PDE (32), and thus this definition relies heavily on the path frozen PDE. We will see in Proposition 3.9 below that, when $G$ is uniformly continuous in $\omega$, one can give an equivalent definition using the original PPDE (1).

(iii) Both (Ekren et al. 2016b) and (Ren et al. 2016b) require the uniform continuity of $G$ in $\omega$, which is not required in this paper. We remark that this uniform regularity can be violated even in the semilinear case: $G=\frac{1}{2} \sigma^{2}(t, \omega): \gamma+f(t, \omega, y, z)$.

In the following proposition we state an alternative definition which is equivalent to Definiton 3.7 when the generator $G$ is uniformly continuous in $\omega$. The proof is postponed to the Appendix.

Proposition 3.9 Let Assumption 3.1 hold true, and assume further that $G$ is uniformly continuous in $\omega$. Then $u$ is a pseudo-Markovian $\mathcal{P}_{L}$-viscosity subsolution if and only if, for any $(t, \omega) \in[0, T) \times \Omega$, there exist $u^{t, \omega, \varepsilon} \in \mathcal{M}_{\varepsilon}\left(\Lambda^{t}\right), \varepsilon>0$, such that

(i) for all $\left(t^{\prime}, \omega^{\prime}\right) \in[t, T) \times \Omega^{t}, u^{t, \omega, \varepsilon}$ is a viscosity subsolution of PPDE (1) at $\left(t^{\prime}, \omega \otimes_{t} \omega^{\prime}\right)$;

(ii) $u^{t, \omega, \varepsilon} \leq u^{t, \omega}$ on $\Lambda^{t}$ and $\lim _{\varepsilon \rightarrow 0} u^{t, \omega, \varepsilon}(t, \boldsymbol{\theta})=u(t, \omega)$. 


\section{Comparison principle}

The main result of this paper is the following comparison principle for pseudoMarkovian Crandall-Lions viscosity solutions. Since a $\mathcal{P}_{L}$-viscosity semisolution is always a Crandall-Lions viscosity semisolution, it also implies the comparison principle for pseudo-Markovian $\mathcal{P}_{L}$-viscosity solutions.

Theorem 4.1 Let Assumption 3.1 hold. Assume $u_{1}$ and $u_{2}$ are pseudo-Markovian Crandall-Lions viscosity subsolution and supersolution of PPDE (1), respectively. If $u_{1}(T, \cdot) \leq u_{2}(T, \cdot)$, then $u_{1} \leq u_{2}$ on $\Lambda$.

Proof In this proof, viscosity semisolutions are always in Crandall-Lions sense. Without loss of generality, we shall only prove $u_{1}(0, \mathbf{0}) \leq u_{2}(0, \mathbf{0})$. For $i=1,2$, let $u_{i}^{\varepsilon} \in \mathcal{M}_{\varepsilon}(\Lambda)$ be the corresponding approximations with corresponding $v_{n}^{i}$. By Definition 3.7 (ii), it suffices to show that $u_{1}^{\varepsilon}(0, \mathbf{0}) \leq u_{2}^{\varepsilon}(0, \mathbf{0})$ for all $\varepsilon>0$. In the rest of this proof we fix $\varepsilon>0$ and denote $w_{n}:=v_{n}^{1}-v_{n}^{2}$.

Step 1 . We first show that, for any $n \geq 0, \pi_{n}=\left(t_{i}, x_{i}\right)_{1 \leq i \leq n} \in \Pi_{n}^{\varepsilon}$, it holds

$$
w_{n}^{+}\left(\pi_{n} ; t_{n}, \mathbf{0}\right) \leq \overline{\mathcal{E}}_{t_{n}}^{L}\left[e^{L\left(\mathrm{H}-t_{n}\right)} w_{n}^{+}\left(\pi_{n} ; \mathrm{H}, B_{\mathrm{H}}^{t_{n}}\right)\right] \quad \text { where } \quad \mathrm{H}:=\mathrm{H}_{1}^{t_{n}, 0, \varepsilon} .
$$

Without loss of generality it is enough to prove the statement for $n=0$. That is, denoting $w:=w_{0}$,

$$
w^{+}(0, \mathbf{0}) \leq \overline{\mathcal{E}}^{L}\left[e^{L \mathrm{H}} w^{+}\left(\mathrm{H}, B_{\mathrm{H}}\right)\right] \quad \text { where } \quad \mathrm{H}:=\mathrm{H}_{1}^{\varepsilon} \text {. }
$$

For any $\delta>0$ small, by Remark 3.6 (ii), $v_{0}^{1}, v_{0}^{2}$ are uniformly continuous viscosity semisolutions of the following PDE:

$$
\partial_{t} v+G\left(t, \mathbf{0}, v, \partial_{x} v, \partial_{x x}^{2} v\right)=0, \quad(t, x) \in D_{1}^{\varepsilon, \delta}
$$

Following the arguments in (Ekren et al. 2016b), Lemma 6.1, or following an alternative argument in (Barles et al. 1997), Lemma 3.7, one can easily prove that for any $(t, x) \in D_{1}^{\varepsilon, \delta}$,

$$
w^{+}(t, x) \leq \overline{\mathcal{E}}_{t}^{L}\left[e^{L\left(\mathrm{H}_{1}^{t, x, \varepsilon-L_{1} t}-t\right)} w^{+}\left(\mathrm{H}_{1}^{t, x, \varepsilon-L_{1} t}, B_{\mathrm{H}_{1}^{t, x, \varepsilon-L_{1} t}}^{t}\right)\right] .
$$

In particular, this implies

$$
w^{+}\left(\delta, \omega_{\delta}\right) \leq \overline{\mathcal{E}}_{\delta}^{L}\left[e^{L\left(\mathrm{H}_{1}^{\delta, x, \varepsilon-L_{1} \delta}-\delta\right)} w^{+}\left(\mathrm{H}_{1}^{\delta, \omega_{\delta}, \varepsilon-L_{1} \delta}, B_{\mathrm{H}_{1}^{\delta, \omega_{\delta}, \varepsilon-L_{1} \delta}}^{\delta}\right)\right], \quad \text { if } \mathrm{H}(\omega)>\delta .
$$

By the uniform regularity of $w$, and using (19) we see that

$$
\overline{\mathcal{E}}^{L}\left[w^{+}\left(\delta, B_{\delta}\right) \mathbf{1}_{\{\mathrm{H}>\delta\}}\right] \leq \overline{\mathcal{E}}^{L}\left[e^{L(\mathrm{H}-\delta)} w^{+}\left(\mathrm{H}, B_{\mathrm{H}}\right) \mathbf{1}_{\{\mathrm{H}>\delta\}}\right] \leq \overline{\mathcal{E}}^{L}\left[e^{L \mathrm{H}} w^{+}\left(\mathrm{H}, B_{\mathrm{H}}\right)\right] .
$$


Then, noting that $|w| \leq C$,

$$
\begin{aligned}
w^{+}(0,0)-\overline{\mathcal{E}}^{L}\left[e^{L \mathrm{H}} w^{+}\left(\mathrm{H}, B_{\mathrm{H}}\right)\right] & \leq w^{+}(0,0)-\overline{\mathcal{E}}^{L}\left[w^{+}\left(\delta, B_{\delta}\right) \mathbf{1}_{\{\mathrm{H}>\delta\}}\right] \\
& \leq \overline{\mathcal{E}}^{L}\left[\left|w^{+}\left(\delta, B_{\delta}\right)-w^{+}(0,0)\right|\right]+C \overline{\mathcal{E}}^{L}\left[\mathbf{1}_{\{\mathrm{H}>\delta\}}\right] \\
& \leq \overline{\mathcal{E}}^{L}\left[\left|w^{+}\left(\delta, B_{\delta}\right)-w^{+}(0,0)\right| \mathbf{1}_{\left\{\left|B_{\delta}\right| \leq \delta^{\frac{1}{3}}\right\}}\right] \\
& +C \overline{\mathcal{E}}^{L}\left[\mathbf{1}_{\left\{\left|B_{\delta}\right|>\delta^{\frac{1}{3}}\right\}}\right]+C \overline{\mathcal{E}}^{L}\left[\mathbf{1}_{\{\mathrm{H}>\delta\}}\right] \rightarrow 0, \text { as } \delta \rightarrow 0,
\end{aligned}
$$

where the first convergence is due to the continuity of $w^{+}$at $(0,0)$, the second one is due to standard estimates, and the third one is due to (20).

Step 2. We next show that, for any $n \geq 0$,

$$
\overline{\mathcal{E}}^{L}\left[e^{L \mathrm{H}_{n}^{\varepsilon}} w_{n}^{+}\left(\pi_{n}^{\varepsilon}(B) ; \mathrm{H}_{n}^{\varepsilon}, \mathbf{0}\right)\right] \leq \overline{\mathcal{E}}^{L}\left[e^{L \mathrm{H}_{n+1}^{\varepsilon}} w_{n+1}^{+}\left(\pi_{n+1}^{\varepsilon}(B) ; \mathrm{H}_{n+1}^{\varepsilon}, 0\right)\right] .
$$

Indeed, for any $\omega$, by (33) and (29) we have: denoting $\mathrm{H}_{n+1}^{\omega, \varepsilon}:=\left(\mathrm{H}_{n+1}^{\varepsilon}\right)^{\mathrm{H}_{n}^{\varepsilon}(\omega), \omega}$,

$$
\begin{aligned}
& e^{L \mathrm{H}_{n}^{\varepsilon}(\omega)} w_{n}^{+}\left(\pi_{n}^{\varepsilon}(\omega) ; \mathrm{H}_{n}^{\varepsilon}(\omega), \mathbf{0}\right) \\
& \quad \leq \overline{\mathcal{E}}_{\mathrm{H}_{n}^{\varepsilon}(\omega)}^{L}\left[e^{L \mathrm{H}_{n+1}^{\omega, \varepsilon}} w_{n+1}^{+}\left(\pi_{n}^{\varepsilon}(\omega),\left(\mathrm{H}_{n+1}^{\omega, \varepsilon}, B_{\mathrm{H}_{n+1}^{\omega, \varepsilon}}^{\mathrm{H}^{\varepsilon}}\right) ; \mathrm{H}_{n+1}^{\omega, \varepsilon}, 0\right)\right],
\end{aligned}
$$

Now for any $\delta>0$ and $\mathbb{P} \in \mathcal{P}$, by the uniform regularity in Remark 3.6 (ii), it follows from the arguments in (Ekren et al. 2016b) (5.5) that,

$$
\begin{gathered}
e^{L \mathrm{H}_{n}^{\varepsilon}} w_{n}^{+}\left(\pi_{n}^{\varepsilon} ; \mathrm{H}_{n}^{\varepsilon}, \mathbf{0}\right) \mathbf{1}_{\cap_{i=1}^{n}\left\{\mathrm{H}_{i}^{\varepsilon}-\mathrm{H}_{i-1}^{\varepsilon} \geq \delta\right\}} \\
\leq \underset{\mathbb{P}^{\prime} \in \mathcal{P}_{L}\left(\mathbb{P}, \mathrm{H}_{n}^{\varepsilon}\right)}{\operatorname{P}} \mathbb{E}^{\mathbb{P}^{\prime}}\left[e^{L \mathrm{H}_{n+1}^{\varepsilon}} w_{n+1}^{+}\left(\pi_{n+1}^{\varepsilon}(B) ; \mathrm{H}_{n+1}^{\varepsilon}, 0\right) \mathbf{1}_{\cap_{i=1}^{n}\left\{\mathrm{H}_{i}^{\varepsilon}-\mathrm{H}_{i-1}^{\varepsilon} \geq \delta\right\}} \mid \mathcal{F}_{\mathrm{H}_{n}^{\varepsilon}}\right], \quad \mathbb{P} \text {-a.s. }
\end{gathered}
$$

where $\mathcal{P}_{L}\left(\mathbb{P}, \mathrm{H}_{n}^{\varepsilon}\right):=\left\{\mathbb{P}^{\prime} \in \mathcal{P}_{L}: \mathbb{P}=\mathbb{P}^{\prime}\right.$ on $\left.\mathcal{F}_{\mathrm{H}_{n}^{\varepsilon}}\right\}$. This implies

$$
\begin{aligned}
& \overline{\mathcal{E}}^{L}\left[e^{L \mathrm{H}_{n}^{\varepsilon}} w_{n}^{+}\left(\pi_{n}^{\varepsilon}(B) ; \mathrm{H}_{n}^{\varepsilon}, \mathbf{0}\right) \mathbf{1}_{\cap_{i=1}^{n}\left\{\mathrm{H}_{i}^{\varepsilon}-\mathrm{H}_{i-1}^{\varepsilon} \geq \delta\right\}}\right] \\
\leq & \overline{\mathcal{E}}^{L}\left[e^{L \mathrm{H}_{n+1}^{\varepsilon}} w_{n+1}^{+}\left(\pi_{n+1}^{\varepsilon}(B) ; \mathrm{H}_{n+1}^{\varepsilon}, 0\right) \mathbf{1}_{\cap_{i=1}^{n}\left\{\mathrm{H}_{i}^{\varepsilon}-\mathrm{H}_{i-1}^{\varepsilon} \geq \delta\right\}}\right] .
\end{aligned}
$$

Recall (20) and send $\delta \rightarrow 0$, we obtain (35) immediately.

Step 3. Applying Step 2 repeatedly, we have

$$
w^{+}(0,0) \leq \overline{\mathcal{E}}^{L}\left[e^{L \mathrm{H}_{n}^{\varepsilon}} w_{n}^{+}\left(\pi_{n}^{\varepsilon}(B) ; \mathrm{H}_{n}^{\varepsilon}, 0\right)\right] \leq e^{L T} \overline{\mathcal{E}}^{L}\left[w_{n}^{+}\left(\pi_{n}^{\varepsilon}(B) ; \mathrm{H}_{n}^{\varepsilon}, 0\right)\right], \forall n \geq 0 .
$$

Note that $w_{n}^{+}\left(\pi_{n}^{\varepsilon}(B) ; \mathrm{H}_{n}^{\varepsilon}, 0\right)=0$ on $\left\{\mathrm{H}_{n}^{\varepsilon}=T\right\}$. Send $n \rightarrow \infty$ and apply Lemma 3.4, we have

$$
w^{+}(0,0) \leq C e^{L T} \overline{\mathcal{E}}^{L}\left[\mathbf{1}_{\left\{\mathrm{H}_{n}^{\varepsilon}<T\right\}}\right] \rightarrow 0 .
$$

This completes the proof. 


\section{Existence}

In this section, we provide a generic existence result.

\section{A bounding equation}

We first investigate a bounding equation which will be crucial for our existence result:

$$
\begin{gathered}
\overline{\mathbb{L}} \bar{v}(t, x):=\partial_{t} \bar{v}(t, x)+\bar{g}\left(\bar{v}, \partial_{x} \bar{v}, \partial_{x x} \bar{v}\right)=0, \\
\bar{g}(y, z, \gamma):=\frac{1}{2} \sup _{0 \leq \sigma \leq \sqrt{2 L} I_{d}}\left[\sigma^{2}: \gamma\right]+L[|y|+|z|]+C_{0},
\end{gathered}
$$

where the control $\sigma$ takes values in $\mathbb{S}^{d}$. We start with a local result:

Lemma 5.1 Let $t_{0}<T, \varepsilon>0$, and $h \in C_{b}^{0}\left(\partial Q_{t_{0}}^{\varepsilon}\right)$. Then the PDE

$$
\overline{\mathbb{L}} \bar{v}(t, x)=0,(t, x) \in Q_{t_{0}}^{\varepsilon} ; \quad \bar{v}(t, x)=h(t, x),(t, x) \in \partial Q_{t_{0}}^{\varepsilon},
$$

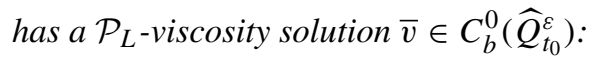

$$
\bar{v}(t, x):=\sup _{b \in \mathbb{L}_{L}^{0}\left(\mathbb{F}^{t}\right)} \overline{\mathcal{E}}_{t}^{L}\left[e^{\int_{t}^{\mathrm{H}^{t, x}} b_{r} d r} h\left(\mathrm{H}^{t, x}, x+B_{\mathrm{H}^{t, x}}^{t}\right)+C_{0} \int_{t}^{\mathrm{H}^{t, x}} e^{\int_{s}^{\mathrm{H}^{t, x}} b_{r} d r} d s\right],
$$

where $\mathrm{H}^{t, x}:=\mathrm{H}^{t, x, \varepsilon-L_{1}\left(t-t_{0}\right)}, \quad \mathbb{L}_{L}^{0}\left(\mathbb{F}^{t}\right):=\left\{b \in \mathbb{L}^{0}\left(\mathbb{F}^{t}\right):|b| \leq L\right\}$.

Proof Assume $t_{0}=0$ for simplicity. First, it is clear that $|\bar{v}| \leq C$. For any $0<$ $\delta<\frac{\varepsilon \wedge T}{L_{1}}$, denote

$$
\bar{Q}_{0}^{\varepsilon, \delta}:=\left\{(t, x) \in \widehat{Q}_{t_{0}}^{\varepsilon}: t \geq \delta\right\}
$$

Note that $\partial Q_{0}^{\varepsilon} \cap \bar{Q}_{0}^{\varepsilon, \delta}$ is compact, then $h$ is uniformly continuous on it. By Lemma 3.2 one may easily show that the $\bar{v}$ defined by (38) is (uniformly) continuous in $\bar{Q}_{0}^{\varepsilon, \delta}$. Provided this regularity, it follows from standard arguments that $\bar{v}$ satisfies the dynamical programming principle, which implies further that $\bar{v}$ is a viscosity solution of PDE (37) in $Q_{0}^{\varepsilon} \cap \bar{Q}_{0}^{\varepsilon, \delta}$. By the arbitrariness of $\delta$, we see that $\bar{v}$ is continuous in $Q_{0}^{\varepsilon} \cap \partial Q_{0}^{\varepsilon}$ and is a viscosity solution of $\operatorname{PDE}(37)$ in $Q_{0}^{\varepsilon}$. Let

It remains to prove the continuity at $(0,0)$. Let $(t, x) \in Q_{0}^{\varepsilon}$ and denote $\delta:=t+|x|$.

$$
\mathrm{H}:=\mathrm{H}^{0,0, \varepsilon}, \quad \tilde{\mathrm{H}}:=\inf \left\{s \geq t:\left|x+B_{s}-B_{t}\right|+L_{1}(s-t) \geq \varepsilon-L_{1} t\right\} \wedge T .
$$

Then one can easily see that

$$
\begin{aligned}
\bar{v}(0,0) & :=\sup _{b \in \mathbb{L}_{L}^{0}(\mathbb{F})} \overline{\mathcal{E}}^{L}\left[e^{\int_{0}^{\mathrm{H}} b_{r} d r} h\left(\mathrm{H}, B_{\mathrm{H}}\right)+C_{0} \int_{0}^{\mathrm{H}} e^{\int_{s}^{\mathrm{H}} b_{r} d r} d s\right] \\
\bar{v}(t, x) & :=\sup _{b \in \mathbb{L}_{L}^{0}(\mathbb{F})} \overline{\mathcal{E}}^{L}\left[e^{\int_{t}^{\tilde{\mathrm{H}}} b_{r} d r} h\left(\tilde{\mathrm{H}}, x+B_{t, \tilde{\mathrm{H}}}\right)+C_{0} \int_{t}^{\tilde{\mathrm{H}}} e^{\int_{s}^{\tilde{\mathrm{H}}} b_{r} d r} d s\right] .
\end{aligned}
$$


Let $\delta_{0}>0$ be a constant which will be specified later and assume $\delta \leq \delta_{0}$. Denote

$$
\begin{aligned}
& \bar{v}^{\prime}(0,0):=\sup _{b \in \mathbb{L}_{L}^{0}(\mathbb{F})} \overline{\mathcal{E}}^{L}\left[e^{\int_{0}^{\mathrm{H}} b_{r} d r} h\left(\mathrm{H} \vee \delta_{0}, B_{\mathrm{H}}\right)+C_{0} \int_{0}^{\mathrm{H}} e^{\int_{s}^{\mathrm{H}} b_{r} d r} d s\right] ; \\
& \bar{v}^{\prime}(t, x):=\sup _{b \in \mathbb{L}_{L}^{0}(\mathbb{F})} \overline{\mathcal{E}}^{L}\left[e^{\int_{t}^{\tilde{\mathrm{H}}} b_{r} d r} h\left(\tilde{\mathrm{H}} \vee \delta_{0}, x+B_{t, \tilde{\mathrm{H}}}\right)+C_{0} \int_{t}^{\tilde{\mathrm{H}}} e^{\int_{s}^{\tilde{\mathrm{H}}} b_{r} d r} d s\right] .
\end{aligned}
$$

Here $h\left(\delta_{0}, x\right):=h\left(\delta_{0}, \frac{\varepsilon-L_{1} \delta_{0}}{|x|} x\right)$ when $|x|>\varepsilon-L_{1} \delta_{0}$. Then, for $\delta \leq \frac{\varepsilon}{2} \wedge \delta_{0}$, by (20) we have

$$
\begin{aligned}
\left|\bar{v}^{\prime}(0,0)-\bar{v}(0,0)\right| & \leq C \sup _{\mathbb{P} \in \mathcal{P}^{L}} \mathbb{P}\left(\mathrm{H} \leq \delta_{0}\right) \leq C_{\varepsilon} \delta_{0} ; \quad\left|\bar{v}^{\prime}(t, x)-\bar{v}(t, x)\right| \\
& \leq C \sup _{\mathbb{P} \in \mathcal{P}^{L}} \mathbb{P}\left(\tilde{\mathrm{H}} \leq \delta_{0}\right) \leq C_{\varepsilon} \delta_{0} .
\end{aligned}
$$

Notice that $h$ is uniformly continuous on $\bar{Q}_{0}^{\varepsilon, \delta_{0}}$, by Lemma 3.2 one can easily prove that

$$
\left|\bar{v}^{\prime}(0,0)-\bar{v}^{\prime}(t, x)\right| \leq C \rho_{\delta_{0}}(\delta),
$$

where the modulus of continuity function $\rho_{\delta_{0}}$ may depend on $\delta_{0}$. Thus, for any $\delta_{0}>0$,

$$
|\bar{v}(0,0)-\bar{v}(t, x)| \leq C \rho_{\delta_{0}}(\delta)+C_{\varepsilon} \delta_{0} .
$$

This implies $\varlimsup_{\delta \rightarrow 0}|\bar{v}(0,0)-\bar{v}(t, x)| \leq C_{\varepsilon} \delta_{0}$. Since $\delta_{0}$ is arbitrary, we obtain $\lim _{\delta \rightarrow 0} \bar{v}(t, x)=\bar{v}(0,0)$.

We next extend the above construction to a global one on $[0, T]$. Our construction is similar to that in (Ekren et al. 2016a), Section "Path dependent Isaacs equation". Given $\left(\pi_{n}, t, x\right) \in D_{n+1}^{\varepsilon}$, define

$$
\mathrm{H}_{1}^{t, x}:=\mathrm{H}^{t, x, \varepsilon-L_{1}\left(t-t_{n}\right)}, \quad \mathrm{H}_{m+1}^{t, x}:=\mathrm{H}^{\mathrm{H}_{m}^{t, x}, 0, \varepsilon}\left(B_{.}^{t}-B_{\mathrm{H}_{m}^{t, x}}^{t}\right), m \geq 1,
$$

and let $B^{\varepsilon, \pi_{n}, t, x}$ be the linear interpolation of: denoting $t_{0}:=0$,

$$
\left(t_{i}, \sum_{j=1}^{i} x_{j}\right)_{0 \leq i \leq n}, \quad\left(\mathrm{H}_{m}^{t, x}, \sum_{i=1}^{n} x_{i}+x+B_{\mathrm{H}_{m}^{t, x}}^{t}\right)_{m \geq 1} .
$$

We then define

$$
\bar{\theta}_{n}^{\varepsilon}\left(\pi_{n} ; t, x\right):=\sup _{b \in \mathbb{L}_{L}^{0}\left(\mathbb{F}^{t}\right)} \overline{\mathcal{E}}^{L}\left[e^{\int_{t}^{T} b_{r} d r} \xi\left(B^{\varepsilon, \pi_{n}, t, x}\right)+C_{0} \int_{t}^{T} e^{\int_{s}^{T} b_{r} d r} d s\right] .
$$

Our main result of this subsection is:

Lemma 5.2 For any $\xi \in U C_{b}(\Omega), \bar{\theta}_{n}^{\varepsilon}$ is bounded and continuous in $D_{n+1}^{\varepsilon}$. 
We remark that in general $\bar{\theta}_{n}^{\varepsilon}$ may not be continuous on the closure of $D_{n+1}^{\varepsilon}$. The proof of Lemma 5.2 relies heavily on the regularity of the hitting times established in Lemma 3.2. It is quite lengthy and we postpone it to Appendix.

\section{A general existence result}

We shall make the following assumption.

Assumption 5.3 For any $\varepsilon>0, \pi_{n} \in \Pi_{n}^{\varepsilon}$, and $h \in C_{b}^{0}\left(\partial Q_{t_{n}}^{\varepsilon}\right)$, the PDE (32) with boundary condition $h$ has a $\mathcal{P}_{L}$-viscosity solution (resp. Crandall-Lions viscosity solution) $v_{n} \in C_{b}^{0}\left(\widehat{Q}_{t_{n}}^{\varepsilon}\right)$.

The following lemma is crucial.

Lemma 5.4 Let Assumptions 3.1 and 5.3 hold. Then there exists $u^{\varepsilon} \in \mathcal{M}_{\varepsilon}(\Lambda)$ with corresponding $\theta_{n}^{\varepsilon}:=v_{n}$ such that

(i) $\theta_{n}^{\varepsilon}\left(\pi_{n} ; \cdot\right)$ is a $\mathcal{P}_{L}$-viscosity solution (resp. Crandall-Lions viscosity solution) to PDE (32);

(ii) $\left.u^{\varepsilon}(T, \omega)=\xi\left(\omega^{\left(\mathrm{H}_{n}^{\varepsilon}, \omega_{\mathrm{H}_{n}^{\varepsilon}}-\omega_{\mathrm{H}_{n-1}^{\varepsilon}}\right.}\right)_{n \geq 1}\right)$.

Proof We follow the arguments in (Ekren et al. 2016b), Lemma 6.3. By Lemmas 5.1 and 5.2 we see that $\bar{\theta}_{n}^{\varepsilon}\left(\pi_{n} ; \cdot\right)$ is a viscosity solution of PDE (37) in $Q_{t_{n}}^{\varepsilon} \cup \partial Q_{t_{n}}^{\varepsilon}$, for any given $\pi_{n} \in \Pi_{n}^{\varepsilon}$. Introduce similarly

$$
\underline{\theta}_{n}^{\varepsilon}\left(\pi_{n} ; t, x\right):=\inf _{b \in \mathbb{L}_{L}^{0}(\mathbb{F})} \underline{\mathcal{E}}^{L}\left[e^{\int_{t}^{T} b_{r} d r} \xi\left(B^{\varepsilon, \pi_{n}, t, x}\right)-C_{0} \int_{t}^{T} e^{\int_{s}^{T} b_{r} d r} d s\right],
$$

which corresponds to the lower bounding equation:

$$
\begin{gathered}
\underline{\mathbb{L} v}(t, x):=\partial_{t} \bar{v}(t, x)+\underline{g}\left(\underline{v}, \partial_{x} \underline{v}, \partial_{x x} \underline{v}\right)=0, \\
\underline{g}(y, z, \gamma):=\frac{1}{2} \inf _{0 \leq \sigma \leq \sqrt{2 L} I_{d}}\left[\sigma^{2}: \gamma\right]-L[|y|+|z|]-C_{0} .
\end{gathered}
$$

For each $m \geq 1$, define two functions on $D_{m+1}^{\varepsilon}$ :

$$
\bar{\theta}_{m}^{\varepsilon, m}\left(\pi_{m} ; t, x\right):=\bar{\theta}_{m}^{\varepsilon}\left(\pi_{m} ; t, x\right), \quad \underline{\theta}_{m}^{\varepsilon, m}\left(\pi_{m} ; t, x\right):=\underline{\theta}_{m}^{\varepsilon}\left(\pi_{m} ; t, x\right) .
$$

We next define $\bar{\theta}_{i}^{\varepsilon, m}\left(\pi_{i} ; \cdot\right) \in C^{0}\left(\widehat{Q}_{t_{i}}^{\varepsilon}\right)$ and $\underline{\theta}_{i}^{\varepsilon, m}\left(\pi_{i} ; \cdot\right) \in C^{0}\left(\widehat{Q}_{t_{i}}^{\varepsilon}\right), i=m-1, \cdots, 0$, backwardly as the unique viscosity solutions of the following PDE:

$$
\partial_{t} v(s, x)+G\left(s, \omega^{\pi_{i}}, v, \partial_{x} v, \partial_{x x}^{2} v\right)=0, \quad(s, x) \in Q_{t_{i}}^{\varepsilon},
$$

with boundary conditions $\bar{\theta}_{i+1}^{\varepsilon, m}\left(\pi_{i},(t, x) ; t, 0\right)$ and $\underline{\theta}_{i+1}^{\varepsilon, m}\left(\pi_{i},(t, x) ; t, 0\right)$, respectively. Here the existence of such viscosity solution is due to Assumption 5.3, while the comparison principle and the uniqueness of viscosity solutions are implied by 
Assumption 3.1. Clearly $\underline{\theta}_{m+1}^{\varepsilon} \leq \bar{\theta}_{m+1}^{\varepsilon}$. Since $\underline{g} \leq G(t, \omega, \cdot) \leq \bar{g}$, by the comparison principle for the PDE (44) we see that

$$
\underline{\theta}_{m}^{\varepsilon, m} \leq \underline{\theta}_{m}^{\varepsilon, m+1} \leq \bar{\theta}_{m}^{\varepsilon, m+1} \leq \bar{\theta}_{m}^{\varepsilon, m} .
$$

Then, we repeatedly apply the comparison principle for the $\operatorname{PDE}(44)$ on $Q_{t_{i}}^{\varepsilon}$, backwardly in $i$, to obtain

$$
\underline{\theta}_{i}^{\varepsilon} \leq \underline{\theta}_{i}^{\varepsilon, m} \leq \underline{\theta}_{i}^{\varepsilon, m+1} \leq \bar{\theta}_{i}^{\varepsilon, m+1} \leq \bar{\theta}_{i}^{\varepsilon, m} \leq \bar{\theta}_{i}^{\varepsilon}, \quad 1 \leq i \leq m .
$$

Denote $w_{i}^{\varepsilon, m}:=\bar{\theta}_{i}^{\varepsilon, m}-\underline{\theta}_{i}^{\varepsilon, m}$ and recall the notations in (39). Since both $\bar{\theta}_{i}^{\varepsilon, m}$ and $\underline{\theta}_{i}^{\varepsilon, m}$ are viscosity solutions of PDE (44) (with different boundary conditions), it follows from the proof of Theorem 4.1, in particular (36), that

$$
\begin{aligned}
& 0 \leq w_{i}^{\varepsilon, m}\left(\pi_{i} ; t, x\right) \\
& \leq C \overline{\mathcal{E}}_{t}^{L}\left[w_{m}^{\varepsilon, m}\left(\pi_{i},\left(\mathrm{H}_{1}^{t, x}, x+B_{\mathrm{H}_{1}}^{t}\right),\left(\mathrm{H}_{i}^{t, x}, B_{\mathrm{H}_{i}^{t, x}}^{t}-B_{\mathrm{H}_{i-1}^{t, x}}^{t}\right)_{2 \leq i \leq m-i} ; \mathrm{H}_{m-i}^{t, x}, 0\right)\right] .
\end{aligned}
$$

It is clear that $w_{m}^{\varepsilon, m}\left(\pi_{m} ; t_{m}, 0\right)=0$ when $t_{m}=T$. Then, by Lemma 3.4,

$$
0 \leq w_{i}^{\varepsilon, m}\left(\pi_{i} ; t, x\right) \leq C \sup _{\mathbb{P} \in \mathcal{P}_{L}^{t}} \mathbb{P}\left(\mathrm{H}_{m-i}^{t, x}<T\right) \leq \frac{C}{(m-i)^{2} \varepsilon^{2}} \rightarrow 0, \quad \text { as } m \rightarrow \infty .
$$

Together with the monotonicity (in $m$ ) in (45), this implies the following limits exist and are equal:

$$
\theta_{n}^{\varepsilon}:=\lim _{m \rightarrow \infty} \bar{\theta}_{n}^{\varepsilon, m}=\lim _{m \rightarrow \infty} \underline{\theta}_{n}^{\varepsilon, m},
$$

where the first limit is decreasing and the second one increasing. Since $\bar{\theta}_{n}^{\varepsilon, m}$ and $\underline{\theta}_{n}^{\varepsilon, m}$ are continuous in $\widehat{D}_{n+1}^{\varepsilon}$, then as their monotone limit $\theta_{n}^{\varepsilon}$ is both upper semicontinuous and lower semicontinuous, and consequently $\theta_{n}^{\varepsilon}$ is also continuous in $\widehat{D}_{n+1}^{\varepsilon}$. The viscosity property of $\theta_{n}^{\varepsilon}$ follows from the standard stability result, and all other properties can be straightforwardly verified.

Our main existence result is as follows.

Theorem 5.5 Let Assumptions 3.1 and 5.3 hold, and $u^{\varepsilon}$ be as in Lemma 5.4. If $u^{\varepsilon}$ converges to $u$ uniformly, then $u$ is a pseudo-Markovian $\mathcal{P}_{L}$-viscosity solution (resp. Crandall-Lions viscosity solution) of PPDE (1) with terminal condition $\xi$.

Proof Without loss of generality, we may assume $G$ is non-increasing in $y$. Denote

$$
\begin{aligned}
\rho(\varepsilon) & :=\left\|u^{\varepsilon}-u\right\|_{\infty}, \theta_{n}^{\varepsilon,+}:=\theta_{n}^{\varepsilon}+\rho(\varepsilon), u^{\varepsilon,+} \\
& :=u^{\varepsilon}+\rho(\varepsilon), \theta_{n}^{\varepsilon,-}:=\theta_{n}^{\varepsilon}-\rho(\varepsilon), u^{\varepsilon,-}:=u^{\varepsilon}-\rho(\varepsilon) .
\end{aligned}
$$

Then $u^{\varepsilon,-} \leq u \leq u^{\varepsilon,+}$, and $\lim _{\varepsilon \rightarrow 0} u^{\varepsilon,+}=u=\lim _{\varepsilon \rightarrow 0} u^{\varepsilon,-}$. It is straightforward to show that $\theta_{n}^{\varepsilon, \overline{+}}\left(\pi_{n} ; \cdot\right)$ and $\theta_{n}^{\varepsilon,-}\left(\pi_{n} ; \cdot\right)$ are viscosity supersolution and viscosity subsolution of PDE (32), respectively. This implies that $u$ is a pseudo-Markovian viscosity solution of PPDE (1). Finally, it is obvious that $u(T, \cdot)=\xi$. 


\section{Stochastic HJB equations}

The stochastic HJB equation is introduced in (Peng 1992) to characterize the value function for an optimization problem with random coefficients. Let $\mathbb{U}$ be an arbitrary measurable set, $\mathcal{U}^{t}$ be the set of $\mathbb{F}^{t}$-progressively measurable and $\mathbb{U}$-valued processes. Given $(t, \omega, x) \in[0, T) \times \Omega \times \mathbb{R}^{d^{\prime}}$ for some dimension $d^{\prime}$ and $\alpha \in \mathcal{U}^{t}$, consider the following controlled decoupled FBSDE:

$$
\begin{aligned}
& X_{s}=x+\int_{t}^{s} b^{t, \omega}\left(r, B^{t}, X_{r}, \alpha_{r}\right) d r+\int_{t}^{s} \sigma^{t, \omega}\left(r, B^{t}, X_{r}, \alpha_{r}\right) d B_{r}^{t} ; \\
& Y_{s}=g^{t, \omega}\left(B^{t}, X_{T}\right)+\int_{s}^{T} f^{t, \omega}\left(r, B^{t}, X_{r}, Y_{r}, Z_{r}, \alpha_{r}\right) d r-\int_{s}^{T} Z_{r} d B_{r} ;
\end{aligned} \quad t \leq s \leq T, \mathbb{P}_{0}^{t} \text {-a.s. }
$$

Here $Y$ is a scalar process. $b, \sigma, f$, and $g$ have appropriate dimensions. $b, \sigma$, and $f$ are $\mathbb{F}$-progressively measurable in all variables, and $g$ is $\mathcal{F}_{T} \times \mathcal{B}\left(\mathbb{R}^{d^{\prime}}\right)$-measurable. We shall assume

Assumption 6.1 (i) $b(t, \omega, x, \alpha), \sigma(t, \omega, x, \alpha), f(t, \omega, x, 0,0, \alpha)$, and $g(\omega, x)$ are bounded;

(ii) $b$ and $\sigma$ are uniformly Lipschitz continuous in $x$, and $f$ is uniformly Lipschitz continuous in $(y, z)$;

(iii) $b$ and $\sigma$ are uniformly continuous in $\omega$; and $f$ and $g$ are uniformly continuous in $(\omega, x)$;

(iv) $b, \sigma$ and $f$ are continuous in $t$.

Under the above conditions, it is clear that the decoupled FBSDE (46) has a unique solution, denoted as $\left(X^{t, \omega, x, \alpha}, Y^{t, \omega, x, \alpha}, Z^{t, \omega, x, \alpha}\right)$. We now introduce the optimization problem:

$$
u^{0}(t, \omega, x):=\sup _{\alpha \in \mathcal{U}^{t}} Y_{t}^{t, \omega, x, \alpha} .
$$

To characterize the above random field $u^{0}$, (Peng 1992) introduced the following stochastic HJB equation (in a simpler case) with $\mathbb{F}$-progressively measurable solution pair $(u, q)$ :

$$
\begin{aligned}
d u(t, \omega, x)= & -\sup _{\alpha \in \mathbb{U}}\left[\frac{1}{2} \partial_{x x} u: \sigma \sigma^{\top}(t, \omega, x, \alpha)+\partial_{x} q: \sigma(t, \omega, x, \alpha)\right. \\
& \left.+\partial_{x} u b(t, \omega, x, \alpha)+f\left(t, \omega, x, u, q+\partial_{x} u \sigma(t, \omega, x, \alpha), \alpha\right)\right]+q(t, \omega, x) d B_{t} \\
u(T, \omega, x)= & g(\omega, x) .
\end{aligned}
$$

This is a special type of backward SPDE. When $\sigma$ does not depend on $\alpha$, namely there is no diffusion control, (Peng 1992) established its wellposedness in Sobolev solutions sense. The recent work (Qiu 2016) extended the result to the general case with diffusion control, also in terms of Sobolev solutions. 
We shall view the Eq. (48) as a PPDE, as shown in (Ekren et al. 2016a), Section "Stochastic HJB equations":

$$
\begin{aligned}
\mathcal{L} u(t, \omega, x)= & 0, \quad u(T, \omega, x)=g(\omega, x), \quad \text { where } \\
\mathcal{L} u(t, \omega, x):= & \partial_{t} u+\frac{1}{2} \operatorname{tr}\left(\partial_{\omega \omega} u\right)+\sup _{\alpha \in \mathbb{U}}\left[\frac{1}{2} \partial_{x x} u: \sigma^{2}(t, \omega, x, \alpha)+\partial_{x \omega} u: \sigma(t, \omega, x, \alpha)\right. \\
& \left.+\partial_{x} u b(t, \omega, x, \alpha)+f\left(t, \omega, x, u, \partial_{\omega} u+\partial_{x} u \sigma(t, \omega, x, \alpha), \alpha\right)\right] .
\end{aligned}
$$

Indeed, if $u$ is smooth, by comparing (48) and the functional Itô formula (5) and noting that $d\langle B\rangle_{t}=I_{d} d t, \mathbb{P}_{0}$-a.s., one may obtain $q=\partial_{\omega} u$ and then (49) follows immediately. In general, of course, $u^{0}$ is not smooth. Our goal is to characterize $u^{0}$ as the unique pseudo-Markovian viscosity solution of (49). We remark that, the Sobolev theory in (Peng 1992, Qiu 2016) requires the special structure of HJB equation, and may not be easy to extend to more general cases like Isaacs equations induced from games. Our viscosity solution characterization, however, can easily be extended as we will see in next section.

The PPDE (48) is slightly different from (1) due to the involvement of the additional variable $x$. In (Ekren et al. 2016a) we view $x$ as the current value of another path $\tilde{\omega}$, namely we increase the dimension of the canonical space with canonical paths $(\omega, \tilde{\omega})$, and consider only viscosity solutions in the form $u\left(t, \omega\right.$. $\left.\tilde{\omega}_{t}\right)$. We emphasize that this PPDE is always degenerate, and thus is not covered by the comparison result in (Ekren et al. 2016b). The results in this paper apply to this case, and $u^{0}$ is indeed the unique pseudo-Markovian viscosity solution of PPDE (49).

However, in this subsection we shall treat (49) in an alternative way. Note that the main feature of our new notion is the Markovian structure. Since the $x$ part is already Markovian, there is no need to introduce an additional path $\tilde{\omega}$ and then discretize it. So we shall discretize $\omega$ only and deal with $x$ directly. For notational clarity, we will use $\bar{x}$ to denote the variable induced from the discretization of $\omega$. Recall the notations in (25) and (27) corresponding to the discretization of $\omega$. Analogous to Definitions 3.5 and 3.7, we define:

Definition 6.2 Let $\varepsilon>0$. We say $u \in \mathbb{L}^{0}\left(\Lambda \times \mathbb{R}^{d^{\prime}}\right)$ is $\varepsilon$-Markovian, denoted as $u \in \mathcal{M}_{\varepsilon}\left(\Lambda \times \mathbb{R}^{d^{\prime}}\right)$, if there exist deterministic functions $v_{n}: D_{n+1}^{\varepsilon} \times \mathbb{R}^{d^{\prime}} \rightarrow \mathbb{R}$, $n \geq 0$, satisfying:

(i) (2) holds, namely

$$
\begin{aligned}
u(t, \omega, x)= & \sum_{n=0}^{\infty} v_{n}\left(\pi_{n}^{\varepsilon}(\omega) ; t, \omega_{t}-\omega_{\mathrm{H}_{n}^{\varepsilon}(\omega)}, x\right) \\
& \mathbf{1}_{\left\{\mathrm{H}_{n}^{\varepsilon}(\omega) \leq t<\mathrm{H}_{n+1}^{\varepsilon}(\omega) \text { or } \mathrm{H}_{n}^{\varepsilon}(\omega)<\mathrm{H}_{n+1}^{\varepsilon}(\omega)=T=t\right\}} \cdot .
\end{aligned}
$$

(ii) For all $\pi_{n}=\left(t_{i}, x_{i}\right)_{1 \leq i \leq n} \in \Pi_{n}^{\varepsilon}$ and $(t, \bar{x}, x) \in \partial Q_{t_{n}}^{\varepsilon} \times \mathbb{R}^{d^{\prime}}$, the following compatibility condition holds

$$
v_{n}\left(\pi_{n} ; t, \bar{x}, x\right)=v_{n+1}\left(\pi_{n},(t, \bar{x}) ; t, 0, x\right) .
$$


(iii) Each $v_{n}, n \geq 0$, is continuous in $D_{n+1}^{\varepsilon} \times \mathbb{R}^{d^{\prime}}$.

Definition 6.3 We say $u$ is a pseudo-Markovian $\mathcal{P}_{L}$-viscosity subsolution (resp. Crandall-Lions viscosity subsolution) of PPDE (49) at $(t, \omega, x) \in[0, T) \times \Omega \times \mathbb{R}^{d^{\prime}}$ if there exist $u^{t, \omega, \varepsilon} \in \mathcal{M}_{\varepsilon}\left(\Lambda^{t} \times \mathbb{R}^{d^{\prime}}\right)$ with corresponding $\left\{v_{n}, n \geq 1\right\}, \varepsilon>0$, such that

(i) for each $\pi_{n}=\left(t_{i}, x_{i}\right)_{1 \leq i \leq n} \in \Pi_{n}^{t, \varepsilon}, v_{n}\left(\pi_{n} ; \cdot\right)$ is a $\mathcal{P}_{L}$-viscosity subsolution (resp. Crandall-Lions viscosity subsolution) to the following PDE: at $\left(\pi_{n}, s, \bar{x}, x\right) \in \Pi_{n}^{t, \varepsilon} \times Q_{t_{n}}^{\varepsilon} \times \mathbb{R}^{d^{\prime}}$,

$$
\begin{aligned}
\mathbb{L}^{t, \omega, \pi_{n}} v_{n}:=\quad \partial_{t} v_{n}+\frac{1}{2} \partial_{\bar{x} \bar{x}}^{2} v_{n}: I_{d}+\sup _{\alpha \in \mathbb{U}}\left[\frac{1}{2} \partial_{x x} u: \sigma^{2}\left(t, \omega \otimes_{t} \omega^{\pi_{n}}, x, \alpha\right)+\partial_{x \bar{x}} u: \sigma\left(t, \omega \otimes_{t} \omega^{\pi_{n}}, x, \alpha\right)\right. \\
\left.+\partial_{x} u b\left(t, \omega \otimes_{t} \omega^{\pi_{n}}, x, \alpha\right)+f\left(t, \omega \otimes_{t} \omega^{\pi_{n}}, x, u, \partial_{\bar{x}} u, \partial_{x} u, \alpha\right)\right]=0 .
\end{aligned}
$$

where $\omega^{\pi_{n}}$ is the linear interpolation of $(t, \boldsymbol{0}),\left(t_{i}, \sum_{j=1}^{i} x_{j}\right)_{1 \leq i \leq n},\left(T, \sum_{j=1}^{n} x_{j}\right)$, (ii) $u^{t, \omega, \varepsilon} \leq u^{t, \omega}$ on $\Lambda^{t} \times \mathbb{R}^{d^{\prime}}$ and $\lim _{\mathcal{E} \rightarrow 0} u^{t, \omega, \varepsilon}(t, \boldsymbol{0}, x)=u(t, \omega, x)$.

We define a pseudo-Markovian viscosity supersolution similarly, and we call $u$ a pseudo-Markovian viscosity solution if it is both a pseudo-Markovian viscosity subsolution and supersolution.

Our main result of this section is:

Theorem 6.4 Let Assumption 6.1 hold and $L>0$ be large enough. Then the $u^{0}$ defined by (47) is the unique pseudo-Markovian viscosity solution (both in $\mathcal{P}_{L}$-sense and in Crandall-Lions sense) of PPDE (49).

Proof Clearly Assumption 6.1 implies Assumption 3.1, with the state space raised to $(\omega, x)$ due to the involvement of $x$. Then the comparison principle follows the same arguments as in Theorem 4.1, which implies the uniqueness immediately. So it suffices to verify that $u^{0}$ is indeed a pseudo-Markovian viscosity solution. Without loss of generality, we shall only verify the viscosity property at $(0,0)$. We note that, due to the representation (47), we shall construct the functions $\theta_{n}^{\varepsilon}$ in Lemma 5.4 directly, without referring to Assumption 5.3. We next verify the uniform convergence of the corresponding $u^{\varepsilon}$, and then the existence follows from Theorem 5.5.

Our construction of the functions $\theta_{n}^{\varepsilon}$ is similar to that in Subsection "A bounding equation". Fix $\varepsilon>0$, and let $\left(\pi_{n}, t, \bar{x}, x\right) \in D_{n+1}^{\varepsilon} \times \mathbb{R}^{d^{\prime}}$. Recall the notations in (39) and (40), and denote

$$
\mathrm{H}_{0}:=t_{n}, \quad \mathrm{H}_{m}:=\mathrm{H}_{m}^{t, \bar{x}}, m \geq 1, \quad \widehat{B}:=B^{\varepsilon, \pi_{n}, t, \bar{x}} .
$$

We then define

$$
\theta_{n}^{\varepsilon}\left(\pi_{n} ; t, \bar{x}, x\right):=\sup _{\alpha \in \mathcal{U}^{t}} Y_{t}^{\pi_{n}, t, \bar{x}, x, \alpha},
$$


where $\left(X^{\pi_{n}, t, \bar{x}, x, \alpha}, Y^{\pi_{n}, t, \bar{x}, x, \alpha}, Z^{\pi_{n}, t, \bar{x}, x, \alpha}\right)$ is the solution to the following decoupled FBSDE on $[t, T]$ :

$$
\begin{aligned}
& X_{s}=x+\int_{t}^{s} \sum_{m=0}^{\infty}\left[b\left(r, \widehat{B} \cdot \wedge \mathrm{H}_{m}, X_{r}, \alpha_{r}\right) \mathbf{1}_{\left[\mathrm{H}_{m}, \mathrm{H}_{m+1}\right)} d r+\sigma\left(r, \widehat{B}_{\wedge} \wedge \mathrm{H}_{m}, X_{r}, \alpha_{r}\right) \mathbf{1}_{\left[\mathrm{H}_{m}, \mathrm{H}_{m+1}\right)} d B_{r}^{t}\right] \\
& Y_{s}=g\left(\widehat{B}, X_{T}\right)+\int_{s}^{T} \sum_{m=0}^{\infty} f\left(r, \widehat{B}_{\cdot \wedge \mathrm{H}_{m}}, X_{r}, Y_{r}, Z_{r}, \alpha_{r}\right) \mathbf{1}_{\left[\mathrm{H}_{m}, \mathrm{H}_{m+1}\right)} d r-\int_{s}^{T} Z_{r} d B_{r}, \quad \mathbb{P}_{0}^{t} \text {-a.s. }
\end{aligned}
$$

While it is not completely trivial, it follows from similar arguments in Lemmas 5.1 and 5.2 that $\theta_{n}^{\varepsilon}$ satisfies all the requirements in Lemma 5.4. We leave the details to interested readers.

Moreover, as in (50) we denote

$$
\begin{aligned}
u^{\varepsilon}(t, \omega, x):= & \sum_{n=0}^{\infty} \theta_{n}^{\varepsilon}\left(\pi_{n}^{\varepsilon}(\omega) ; t, \omega_{t}-\omega_{\mathrm{H}_{n}^{\varepsilon}(\omega)}, x\right) \\
& \mathbf{1}_{\left\{\mathrm{H}_{n}^{\varepsilon}(\omega) \leq t<\mathrm{H}_{n+1}^{\varepsilon}(\omega) \text { or } \mathrm{H}_{n}^{\varepsilon}(\omega)<\mathrm{H}_{n+1}^{\varepsilon}(\omega)=T=t\right\}} .
\end{aligned}
$$

It remains to verify that $u^{\varepsilon}$ converges to $u^{0}$ uniformly. Indeed, for any $(t, \omega, x)$ and $\varepsilon>0$, fix the $n$ such that $\mathrm{H}_{n}^{\varepsilon}(\omega) \leq t<\mathrm{H}_{n+1}^{\varepsilon}(\omega)$. For $\pi_{n}:=\pi_{n}^{\varepsilon}(\omega)$, we have

$$
\begin{gathered}
\sup _{t \leq r \leq T}\left\|\omega \otimes_{t} B^{t}-\sum_{m=0}^{\infty} \widehat{B} \cdot \wedge \mathrm{H}_{m} \mathbf{1}_{\left[\mathrm{H}_{m}, \mathrm{H}_{m+1}\right)}(r)\right\|_{r}=\sup _{m \geq 0} \sup _{m \vee t \leq r \leq \mathrm{H}_{m+1}} \\
\left\|\omega \otimes_{t} B^{t}-\widehat{B} \cdot \wedge \mathrm{H}_{m}\right\|_{r} \leq \varepsilon .
\end{gathered}
$$

Since, $b$ and $\sigma$ are uniformly continuous in $\omega$, by standard SDE arguments we have

$$
\mathbb{E}^{\mathbb{P}_{0}^{t}}\left[\left\|X^{\pi_{n}, t, \bar{x}, x, \alpha}-X^{t, \omega, x, \alpha}\right\|_{T}^{2}\right] \leq C \rho_{1}(\varepsilon),
$$

for some modulus of continuity function $\rho_{1}$. Moreover, since $f$ and $g$ are uniformly continuous in $(\omega, x)$, by standard BSDE arguments we obtain

$$
\mathbb{E}^{\mathbb{P}_{0}^{t}}\left[\left\|Y^{\pi_{n}, t, \bar{x}, x, \alpha}-Y^{t, \omega, x, \alpha}\right\|_{T}^{2}+\int_{t}^{T}\left|Z_{r}^{\pi_{n}, t, \bar{x}, x, \alpha}-Z_{r}^{t, \omega, x, \alpha}\right|^{2} d r\right] \leq C \rho_{2}(\varepsilon),
$$

for some modulus of continuity function $\rho_{2}$. By the arbitrariness of $\alpha$, this implies that

$$
\left|u^{\varepsilon}(t, \omega, x)-u^{0}(t, \omega, x)\right| \leq C \rho_{2}(\varepsilon)
$$

Now by Theorem 5.5 we see that $u^{0}$ is a pseudo-Markovian viscosity solution of PPDE (49). 


\section{Path dependent Isaacs equation}

In this section, we study the path-dependent Isaacs equation, which is the PPDE (1) with generator:

$$
\begin{aligned}
& G(t, \omega, y, z, \gamma) \\
& \quad:=\inf _{\beta \in \mathbb{V}} \sup _{\alpha \in \mathbb{U}}\left[\frac{1}{2} \sigma \sigma^{\top}(t, \omega, \alpha, \beta): \gamma+f(t, \omega, y, z \sigma(t, \omega, \alpha, \beta), \alpha, \beta)\right],
\end{aligned}
$$

where $\mathbb{U}$ and $\mathbb{V}$ are two measurable sets, and $\sigma$ and $f$ are $\mathbb{F}$-progressively measurable. We shall make the following assumption.

Assumption 7.1 $(i) \sigma(t, \omega, \alpha, \beta), f(t, \omega, 0,0, \alpha, \beta)$, and $\xi$ are bounded;

(ii) $\sigma$ is uniformly Lipschitz continuous in $\omega$, and $f$ is uniformly Lipschitz. continuous in $(y, z)$;

(iii) $f$ and $\xi$ are uniformly continuous in $\omega$;

(iv) $\sigma$ and $f$ are continuous in $t$.

Under Assumption 7.1, clearly $G$ satisfies Assumption 3.1. Then it follows from Theorem 4.1 that the path-dependent Isaacs Eq. (1)-(57) has at most one pseudo-Markovian viscosity solution. We remark that (Pham and Zhang 2014) established the comparison principle for viscosity solutions of this PPDE in the sense of Definition 2.2. However, it followed the approach in (Ekren et al. 2016b) and requires that: (i) $\sigma$ is uniformly non-degenerate; (ii) $\sigma$ does not depend on $\omega$, and (iii) the dimension $d \leq 2$. None of these additional assumptions is needed in this paper.

The goal of this section is to construct a pseudo-Markovian viscosity solution. It is well known that Isaacs equation is induced from zero-sum stochastic differential games. There are three possible formulations for the game problem: (i) control versus control in strong formulation; (ii) strategy versus control in strong formulation; and (iii) control versus control in weak formulation. We refer to (Pham and Zhang 2014) for detailed discussions on the three formulations. In particular, under the first approach the value function does not satisfy the dynamic programming principle and thus does not provide a representation for the PPDE. As discussed in (Pham and Zhang 2014), the weak formulation in (iii) has some advantages from practical point of view. However, when following this approach, it is more difficult to obtain the desired regularity of the value function. Since our focus here is not the game problem, but to provide a representation for the solution to the PPDE, we shall use the strong formulation (ii) which is easier for regularity. This approach was initiated by (Fleming and Souganidis 1989) for PDEs.

To this end, let $\mathcal{U}^{t}, \mathcal{V}^{t}$ denote the sets of $\mathbb{F}^{t}$-measurable $\mathbb{U}$-valued, $\mathbb{V}$-valued processes, respectively, and $\mathcal{B}^{t}$ the set of adapted strategies $\lambda: \mathcal{V}^{t} \rightarrow \mathcal{U}^{t}$, here adaptedness means: for any $\tau \in \mathcal{T}^{t}$,

$$
\text { if } v^{1}, v^{2} \in \mathcal{V}^{t} \text { such that } v_{s}^{1}=v_{s}^{2}, t \leq s \leq \tau, \quad \text { then } \lambda\left(v^{1}\right)_{s}=\lambda\left(v^{2}\right)_{s}, t \leq s \leq \tau \text {. }
$$


For any $(t, \omega) \in \Omega$ and $(\alpha, \beta) \in \mathcal{U}^{t} \times \mathcal{V}^{t}$, consider the following path-dependent decoupled FBSDEs:

$$
\begin{aligned}
& X_{s}=\omega_{t}+\int_{t}^{s} \sigma^{t, \omega}\left(r, X ., \alpha_{r}, \beta_{r}\right) d B_{r}^{t}, \\
& Y_{s}=\xi^{t, \omega}(X .)+\int_{s}^{T} f^{t, \omega}\left(r, X ., Y_{r}, Z_{r}, \alpha_{r}, \beta_{r}\right) d r-\int_{s}^{T} Z_{r} d B_{r}^{t}, \quad t \leq s \leq T, \quad \mathbb{P}_{0}^{t} \text {-a.s. }
\end{aligned}
$$

Under Assumption 7.1, clearly the above FBSDE is wellposed, and we shall denote its unique solution as $\left(X^{t, \omega, \alpha, \beta}, Y^{t, \omega, \alpha, \beta}, Z^{t, \omega, \alpha, \beta}\right)$. We then define

$$
u^{0}(t, \omega):=\sup _{\lambda \in \mathcal{B}^{t}} \inf _{\beta \in \mathcal{V}^{t}} Y_{t}^{t, \omega, \lambda(\beta), \beta}
$$

Remark 7.2 In (60), the controls $(\lambda(\beta), \beta)$ do not depend on the variable $\omega$, due to its strong formulation. Then, given $\left(t, \omega^{i}\right), i=1,2$, we have

$$
\left|u^{0}\left(t, \omega^{1}\right)-u^{0}\left(t, \omega^{2}\right)\right| \leq \sup _{\lambda \in \mathcal{B}^{t}} \sup _{\beta \in \mathcal{V}^{t}}\left|Y_{t}^{t, \omega^{1}, \lambda(\beta), \beta}-Y_{t}^{t, \omega^{2}, \lambda(\beta), \beta}\right|,
$$

and thus the regularity of $u^{0}$ (in $\omega$ ) follows from standard SDE/BSDE estimates. Under the weak formulation in (Pham and Zhang 2014), the controls $\alpha, \beta$ are feedback type and thus depend on $\omega^{1}, \omega^{2}$. Then we don't have a simple estimate like (61), and the regularity of $u^{0}$ is indeed more difficult to establish.

Our main result of this section is:

Theorem 7.3 Under Assumption 7.1, the $u^{0}$ defined by (60) is the unique pseudoMarkovian viscosity solution of PPDE (1)-(57) with terminal condition $\xi$.

Proof Similar to Theorem 6.4, it suffices to construct the desired functions $\theta_{n}^{\varepsilon}$ and show that the corresponding process $u^{\varepsilon}$ converges to $u^{0}$ uniformly. Again, we will only verify the viscosity property at $(0,0)$.

Fix $\varepsilon>0$, and let $\left(\pi_{n}, t, x\right) \in D_{n+1}^{\varepsilon}$. Recall (32) that $\omega^{\pi_{n}}$ denote the linear interpolation of $(0,0),\left(t_{i}, \sum_{j=1}^{i} x_{j}\right)_{1 \leq i \leq n}$, and $\left(T, \sum_{j=1}^{n} x_{j}\right)$. For any $(\alpha, \beta)$, to adapt to the strong formulation, we define $X:=X^{\pi_{n}, t, x, \alpha, \beta}$ and $\mathrm{H}_{m}:=\mathrm{H}_{m}^{\pi_{n}, t, x, \alpha, \beta}$ recursively as follows. First,

$$
\begin{aligned}
& X_{s}^{1}:=\sum_{i=1}^{n} x_{i}+x+\int_{t}^{s} \sigma\left(r, \omega^{\pi_{n}}, \alpha_{r}, \beta_{r}\right) d B_{r}^{t}, t \leq s \leq T, \mathbb{P}_{0}^{t} \text {-a.s. } \\
& \mathrm{H}_{1}:=\inf \left\{s \geq t:\left|X_{s}^{1}-\sum_{i=1}^{n} x_{i}\right|+L_{1}(s-t) \geq \varepsilon-L_{1}\left(t-t_{n}\right)\right\} \wedge T \\
& X_{s}:=X_{s}^{1}, t \leq s \leq \mathrm{H}_{1} .
\end{aligned}
$$


Next, for $m \geq 1$,

$$
\begin{aligned}
& \widehat{X}^{m}:= \text { linear interpolation of }(0,0),\left(t_{i}, \sum_{j=1}^{i} x_{j}\right)_{1 \leq i \leq n}, \\
&\left(\mathrm{H}_{i}, X_{\mathrm{H}_{i}}\right)_{1 \leq i \leq m},\left(T, X_{\mathrm{H}_{m}}\right) ; \\
& X_{s}^{m+1}:=X_{\mathrm{H}_{m}}+\int_{\mathrm{H}_{m}}^{s} \sigma\left(r, \widehat{X}^{m}, \alpha_{r}, \beta_{r}\right) d B_{r}^{t}, \mathrm{H}_{m} \leq s \leq T, \mathbb{P}_{0^{-a} . \mathrm{s} .} \\
& \mathrm{H}_{m+1}:=\inf \left\{s \geq \mathrm{H}_{m}:\left|X_{s}^{m+1}-X_{\mathrm{H}_{m}}\right|+L_{1}\left(s-\mathrm{H}_{m}\right) \geq \varepsilon\right\} \wedge T ; \\
& X_{s}:=X_{s}^{m+1}, \mathrm{H}_{m} \leq s \leq \mathrm{H}_{m+1} .
\end{aligned}
$$

Denote $\mathrm{H}_{0}:=t_{n}, \widehat{X}:=\lim _{m \rightarrow \infty} X^{m}$, and let $\left(Y^{\pi_{n}, t, x, \alpha, \beta}, Z^{\pi_{n}, t, x, \alpha, \beta}\right)$ be the unique solution of the following BSDE:

$$
\begin{gathered}
Y_{s}=\xi(\widehat{X})+\int_{s}^{T} \sum_{m=0}^{\infty} f\left(r, \widehat{X} \cdot \wedge \mathrm{H}_{m}, Y_{r}, Z_{r}, \alpha_{r}, \beta_{r}\right) \\
\mathbf{1}_{\left[\mathrm{H}_{m}, \mathrm{H}_{m+1}\right)} d r-\int_{s}^{T} Z_{r} d B_{r}^{t}, \quad \mathbb{P}_{0}^{t} \text {-a.s. }
\end{gathered}
$$

We then define

$$
\theta_{n}^{\varepsilon}\left(\pi_{n} ; t, x\right):=\sup _{\lambda \in \mathcal{B}^{t}} \inf _{\beta \in \mathcal{V}^{t}} Y_{t}^{\pi_{n}, t, x, \lambda(\beta), \beta}
$$

In the spirit of (61), combined with standard SDE/BSDE estimates, one may follow the arguments in Lemma 5.2 to show that $\theta_{n}^{\varepsilon} \in C_{b}^{0}\left(D_{n+1}^{\varepsilon}\right)$. Moreover, provided the above regularity and by standard arguments, see e.g. (Fleming and Souganidis 1989), one can prove the dynamic programming principle for $\theta_{n}^{\varepsilon}\left(\pi_{n} ; \cdot\right)$, which leads to the desired viscosity property immediately. We again leave the details to interested readers.

Finally we prove the convergence of $u_{0}^{\varepsilon}=\theta_{0}^{\varepsilon}(0,0)$ with uniform rate. That is, in (62) and (63) we shall set $n=0$ and $(t, x)=(0,0)$. Then we have

$$
X_{s}=\int_{0}^{s} \sum_{m=0}^{\infty} \sigma\left(r, \widehat{X}_{\cdot \wedge \mathrm{H}_{m}}, \alpha_{r}, \beta_{r}\right) \mathbf{1}_{\left[\mathrm{H}_{m}, \mathrm{H}_{m+1}\right)}(r) d B_{r}, 0 \leq s \leq T, \mathbb{P}_{0} \text {-a.s. }
$$

and, by the construction of the hitting times $\mathrm{H}_{m}$,

$$
\|X-\widehat{X}\|_{T} \leq \varepsilon
$$

Compare (66) with the SDE of $X^{0,0, \alpha, \beta}$ in (59), it follows from standard SDE arguments that

$$
\mathbb{E}^{\mathbb{P}_{0}}\left[\left\|X-X^{0,0, \alpha, \beta}\right\|_{T}^{2}\right] \leq C \mathbb{E}^{\mathbb{P}_{0}}\left[\|X-\widehat{X}\|_{T}^{2}\right] \leq C \varepsilon^{2} .
$$


Moreover, let $\rho$ denote the modulus of continuity function of $f$ and $\xi$ in terms of $\omega$. Then, compare (64) with the BSDE of $Y^{0,0, \alpha, \beta}$ in (59), it follows from standard BSDE arguments that

$$
\begin{aligned}
\mathbb{E}^{\mathbb{P}_{0}\left[\left\|Y-Y^{0,0, \alpha, \beta}\right\|_{T}^{2}\right]} & \leq C \mathbb{E}^{\mathbb{P}_{0}}\left[\rho\left(\left\|\widehat{X}-X^{0,0, \alpha, \beta}\right\|_{T}\right)^{2}\right] \\
& \leq C \mathbb{E}^{\mathbb{P}_{0}}\left[\rho\left(\|\widehat{X}-X\|_{T}+\left\|X-X^{0,0, \alpha, \beta}\right\|_{T}\right)^{2}\right] \leq C \rho^{\prime}(\varepsilon)^{2},
\end{aligned}
$$

for a possibly different modulus of continuity function $\rho^{\prime}$ which does not depend on the controls $(\alpha, \beta)$. This implies that, for the $Y$ corresponding to $(\alpha, \beta)=(\lambda(\beta), \beta)$,

$$
\left|u_{0}^{\varepsilon}-u_{0}^{0}\right| \leq \sup _{\lambda \in \mathcal{B}^{0}} \sup _{\beta \in \mathcal{V}^{0}}\left|Y_{0}-Y_{0}^{0,0, \lambda(\beta), \beta}\right| \leq C \rho^{\prime}(\varepsilon),
$$

which provides the desired convergence of $u_{0}^{\varepsilon}$ and thus completes the proof.

We conclude the section with an application on the zero sum game. Denote by $\mathcal{A}^{0}$ the set of adapted strategies $\lambda: \mathcal{U}^{0} \rightarrow \mathcal{V}^{0}$.

Corollary 7.4 Let Assumption 7.1 hold and assume further the following Isaacs condition:

$$
\begin{aligned}
G(t, \omega, y, z, \gamma) & =\tilde{G}(t, \omega, y, z, \gamma) \\
& :=\sup _{\alpha \in \mathbb{U}} \inf _{\beta \in \mathbb{V}}\left[\frac{1}{2} \sigma^{2}(t, \omega, \alpha, \beta): \gamma+f(t, \omega, y, z \sigma(t, \omega, \alpha, \beta), \alpha, \beta)\right] .
\end{aligned}
$$

Then the value of the stochastic differential game exists, namely

$$
u_{0}^{0}=\tilde{u_{0}^{0}}:=\inf _{\lambda \in \mathcal{A}} \sup _{\alpha \in \mathcal{U}} Y_{0}^{0,0, \alpha, \lambda(\alpha)} .
$$

Proof Define $\tilde{u}^{0}(t, \omega)$ in the same spirit as $\tilde{u}_{0}^{0}$. Following the same arguments as in Theorem 7.3, we see that $\tilde{u}^{0}$ is the unique pseudo-Markovian viscosity solution of the PPDE (1) with generator $\tilde{G}$ and terminal condition $\xi$. Since $G=\tilde{G}$, by the uniqueness of the pseudo-Markovian viscosity solution we obtain $u^{0}=\tilde{u}^{0}$.

\section{Appendix}

\section{Proof of Proposition 3.9}

We shall only prove the equivalence at $(0, \mathbf{0})$. The proof for general $(t, \omega)$ follows the same argument. Let $\rho$ denote the modulus of continuity function of $G$ in terms of $\omega$. Moreover, by the change variable formula in (Ekren et al. 2016a), Proposition 3.14, we may assume without loss of generality that

$$
G \text { is non-increasing in } y \text {. }
$$


We first assume $u$ satisfies the subsolution property stated at Proposition 3.9 at $(0, \mathbf{0})$. Let $u^{\varepsilon} \in \mathcal{M}_{\varepsilon}(\Lambda)$ be the approximation given by this property, with corresponding $\left\{v_{n}: n \geq 0\right\}$. Denote

$$
\begin{aligned}
\tilde{u}^{\varepsilon}(t, \omega) & :=u^{\varepsilon}(t, \omega)-\rho(\varepsilon)[T-t], \\
\tilde{v}_{n}\left(\pi_{n} ; t, x\right) & :=v_{n}\left(\pi_{n} ; t, x\right)-\rho(\varepsilon)[T-t] .
\end{aligned}
$$

One may straightforwardly check that

$$
\tilde{u}^{\varepsilon} \in M_{\varepsilon}(\Lambda) \text { with corresponding } \tilde{v}_{n}, \tilde{u}^{\varepsilon} \leq u \text {, and } \lim _{\varepsilon \rightarrow 0} u^{\varepsilon}(0, \mathbf{0})=u(0, \mathbf{0}) .
$$

Then it remains to prove that $\tilde{v}_{n}\left(\pi_{n} ; \cdot\right)$ is a $\mathcal{P}_{L}$-viscosity subsolution of the PDE (32), which in this case becomes:

$$
\begin{aligned}
\mathbb{L}^{\pi_{n}} \tilde{v}_{n}\left(\pi_{n} ; t, x\right) & :=\partial_{t} \tilde{v}_{n}\left(\pi_{n} ; t, x\right)+G\left(s, \omega^{\pi_{n}}, \tilde{v}_{n}, \partial_{x} \tilde{v}_{n}, \tilde{\partial}_{x x}^{2} v_{n}\right) \\
& =0, \quad(t, x) \in Q_{t_{n}}^{\varepsilon} .
\end{aligned}
$$

To see this, we fix $(t, x) \in Q_{t_{n}}^{\varepsilon}$. For any $\tilde{\varphi} \in \underline{\mathcal{A}}^{L} \tilde{u}^{\varepsilon}\left(t, \omega^{\pi_{n},(t, x)}\right)$, namely

$$
\begin{gathered}
\tilde{\varphi} \in C^{1,2}\left(\Lambda^{t}\right), \quad \tilde{\varphi}(t, \mathbf{0})-\tilde{v}_{n}\left(\pi_{n} ; t, x\right)=0 \\
=\inf _{\tau \in \mathcal{T}^{t}} \mathcal{E}_{t}^{L}\left[\tilde{\varphi}_{\tau \wedge \mathrm{H}}-\tilde{v}_{n}\left(\pi_{n} ; \tau \wedge \mathrm{H}, B_{\tau \wedge \mathrm{H}}\right)\right],
\end{gathered}
$$

where we assume without loss of generality that

$$
\mathrm{H} \leq \mathrm{H}_{\varepsilon}:=\inf \left\{s>t:\left|x+B_{s}^{t}\right|+L_{1}(s-t) \geq \varepsilon\right\} \wedge T .
$$

Now denote $\varphi(s, \omega):=\tilde{\varphi}(s, \omega)+\rho(\varepsilon)[T-s]$. Then obviously $\varphi \in$ $\underline{\mathcal{A}}^{L} u^{\varepsilon}\left(t, \omega^{\pi_{n},(t, x)}\right)$, and thus

$$
\partial_{t} \varphi(t, 0)+G^{t, \omega^{\pi_{n},(t, x)}}\left(t, \mathbf{0}, \varphi, \partial_{\omega} \varphi, \partial_{\omega \omega}^{2} \varphi\right) \geq 0 .
$$

Note that

$$
\begin{aligned}
& \partial_{t} \varphi=\partial_{t} \tilde{\varphi}-\rho(\varepsilon), \quad \partial_{\omega} \varphi=\partial_{\omega} \tilde{\varphi}, \quad \partial_{\omega \omega}^{2} \varphi=\partial_{\omega \omega}^{2} \tilde{\varphi}, \\
& \text { and } \\
& \varphi(t, \mathbf{0})=\tilde{\varphi}(t, \mathbf{0})+\rho(\varepsilon) T .
\end{aligned}
$$

Then, by (73) and evaluating $\varphi, \tilde{\varphi}$ and their derivatives at $(t, \mathbf{0})$, we obtain

$$
\begin{aligned}
& \partial_{t} \tilde{\varphi}(t, \mathbf{0})+G\left(t, \omega^{\pi_{n}}, \tilde{\varphi}, \partial_{\omega} \tilde{\varphi}, \tilde{\partial}_{\omega \omega}^{2} \varphi\right) \\
= & \partial_{t} \varphi(t, \mathbf{0})+\rho(\varepsilon)+G\left(t, \omega^{\pi_{n}}, \varphi-\rho(\varepsilon) T, \partial_{\omega} \varphi, \partial_{\omega \omega}^{2} \varphi\right) \\
\geq & -G\left(t, \omega^{\pi_{n},(t, x)}, \varphi, \partial_{\omega} \varphi, \partial_{\omega \omega}^{2} \varphi\right)+\rho(\varepsilon)+G\left(t, \omega^{\pi_{n}}, \varphi, \partial_{\omega} \varphi, \partial_{\omega \omega}^{2} \varphi\right) \\
\geq & \rho(\varepsilon)-\rho\left(\left\|\omega^{\pi_{n}}-\omega^{\pi_{n},(t, x)}\right\|_{t}\right) \geq 0 .
\end{aligned}
$$

This implies that $\tilde{v}_{n}\left(\pi_{n} ; \cdot\right)$ is an $\mathcal{P}_{L}$-viscosity subsolution of the PDE (75), and thus $u$ is a pseudo-Markovian $\mathcal{P}_{L}$-viscosity subsolution at $(0, \mathbf{0})$.

Similarly, if $u$ is a pseudo-Markovian $\mathcal{P}_{L}$-viscosity subsolution at $(0, \mathbf{0})$ with approximation $u^{\varepsilon} \in \mathcal{M}_{\varepsilon}(\Lambda)$ in Definition 3.7, one can show that $u$ satisfies the property in Proposition 3.9 at $(0, \mathbf{0})$ with approximation $\tilde{u}^{\varepsilon}(t, \omega):=u^{\varepsilon}(t, \omega)-\rho(\varepsilon)[T-t]$. 


\section{Proof of Lemma 5.2}

Our proof here relies heavily on the regularity results in Lemma 3.2. Notice that the time regularity (22) requires a shift of canonical process. To facilitate our proof, we extend the canonical space to $\bar{\Omega}:=\left\{\omega \in C\left([0,2 T], \mathbb{R}^{d}\right): \omega_{0}=0\right\}$, and still denote $B, \mathbb{F}$, and $\mathcal{P}_{L}$, etc. in an obvious sense. Given $\left(\pi_{n}, t, x\right) \in D_{n+1}^{\varepsilon}$, define

$$
\begin{aligned}
\mathrm{H}_{1} & :=\inf \left\{s \geq t:\left|x+B_{s-t}\right|+L_{1}(s-t) \geq \varepsilon-L_{1}\left(t-t_{n}\right)\right\} \wedge T, \\
\mathrm{H}_{m+1} & :=\inf \left\{s \geq \mathrm{H}_{m}:\left|B_{\mathrm{H}_{m}+\left(T-\mathrm{H}_{1}\right), s+\left(T-\mathrm{H}_{1}\right)}\right|+L_{1}\left(s-\mathrm{H}_{m}\right) \geq \varepsilon\right\} \wedge T, \quad m \geq 1 \\
N & :=\inf \left\{m \geq 1: \mathrm{H}_{m}=T\right\} .
\end{aligned}
$$

and let $B_{.}^{\varepsilon}\left(\pi_{n}, t, x\right)$ be the path which is the linear interpolation of

$$
\begin{aligned}
\left(t_{i}, X_{i}\right)_{0 \leq i \leq n}, \quad\left(\mathrm{H}_{m}, X_{n+m}\right)_{m \geq 1}, \quad \text { where } t_{0}:=0 \\
\quad X_{i}:=\sum_{j=1}^{i} x_{j}, 0 \leq i \leq n ; \quad X_{n+m}:=X_{n}+x+B_{\mathrm{H}_{1}-t}+B_{T, T+\mathrm{H}_{m}-\mathrm{H}_{1}}, m \geq 1 .
\end{aligned}
$$

One can easily show that the function $\bar{\theta}_{n}^{\varepsilon}$ defined at (41) also satisfies

$$
\begin{gathered}
\bar{\theta}_{n}^{\varepsilon}\left(\pi_{n} ; t, x\right)=\sup _{b \in \mathbb{L}_{L}^{0}(\mathbb{F})} \overline{\mathcal{E}}^{L}\left[e^{\int_{t}^{T} \bar{b}_{r} d r} \xi\left(B^{\varepsilon}\left(\pi_{n}, t, x\right)\right)+C_{0} \int_{t}^{T} e^{\int_{s}^{T} \bar{b}_{r} d r} d s\right] \\
\text { where } \bar{b}_{r}:=b_{r-t} \mathbf{1}_{\left[t, \mathrm{H}_{1}\right)}(r)+b_{T+r-\mathrm{H}_{1}} \mathbf{1}_{\left[\mathrm{H}_{1}, T\right]}(r)
\end{gathered}
$$

Fix $\left(\pi_{n}, t, x\right) \in D_{n+1}^{\varepsilon}$. For arbitrary $\left(\pi_{n}^{\prime}, t^{\prime}, x^{\prime}\right) \in D_{n+1}^{\varepsilon}$, define $\mathrm{H}_{m}^{\prime}, \bar{b}^{\prime}$, and $N^{\prime}$ in the obvious way. The advantage of (76)-(78) is the fact that under this representation one may easily check that,

$$
\begin{gathered}
\bar{b}_{t+r}=\bar{b}_{t^{\prime}+r}, 0 \leq r \leq\left(\mathrm{H}_{1}-t\right) \wedge\left(\mathrm{H}_{1}^{\prime}-t^{\prime}\right) \\
\bar{b}_{\mathrm{H}_{1}+r}=\bar{b}_{\mathrm{H}_{1}^{\prime}+r}, 0 \leq r \leq\left(T-\mathrm{H}_{1}\right) \wedge\left(T-\mathrm{H}_{1}^{\prime}\right) \\
B_{\mathrm{H}_{1}, \mathrm{H}_{1}+r}^{\varepsilon}\left(\pi_{n}, t, x\right)=B_{\mathrm{H}_{1}^{\prime}, \mathrm{H}_{1}^{\prime}+r}\left(\pi_{n}^{\prime}, t^{\prime}, x^{\prime}\right), r \leq\left(\mathrm{H}_{N-1}-\mathrm{H}_{1}\right) \wedge\left(\mathrm{H}_{N-1}^{\prime}-\mathrm{H}_{1}^{\prime}\right), \\
\mathrm{H}_{m+1}-\mathrm{H}_{m}=\mathrm{H}_{m+1}^{\prime}-\mathrm{H}_{m}^{\prime}, \quad 1 \leq m \leq N \wedge N^{\prime}-2 \\
N^{\prime} \leq N \text { on }\left\{\mathrm{H}_{1} \leq \mathrm{H}_{1}^{\prime}\right\}, \quad N \leq N^{\prime} \text { on }\left\{\mathrm{H}_{1}^{\prime} \leq \mathrm{H}_{1}\right\}
\end{gathered}
$$

Denote

$$
\delta:=\max _{1 \leq i \leq n}\left[\left|t_{i}-t_{i}^{\prime}\right|+\left|x_{i}-x_{i}^{\prime}\right|\right] \bigvee\left[\left|t-t^{\prime}\right|+\left|x-x^{\prime}\right|\right],
$$

and for notational simplicity,

$$
\tilde{\mathrm{H}}_{1}:=\mathrm{H}_{1}-t, \quad \tilde{\mathrm{H}}_{1}^{\prime}:=\mathrm{H}_{1}^{\prime}-t^{\prime}, \quad \tilde{T}:=T-\mathrm{H}_{1}, \quad \tilde{T}^{\prime}:=T-\mathrm{H}_{1}^{\prime} .
$$

Note that $\mathrm{H}_{1}=\left(t+\mathrm{H}^{0, x, \varepsilon-L_{1}\left(t-t_{n}\right)}\right) \wedge T, \mathrm{H}_{1}^{\prime}:=\left(t^{\prime}+\mathrm{H}^{0, x^{\prime}, \varepsilon-L_{1}\left(t^{\prime}-t_{n}^{\prime}\right)}\right) \wedge T$. By Lemma 3.2,

$$
\overline{\mathcal{E}}^{L}\left[\left|\mathrm{H}_{1}-\mathrm{H}_{1}^{\prime}\right|\right] \leq C \delta, \quad \text { which implies } \sup _{\mathbb{P} \in \mathcal{P}_{L}} \mathbb{P}\left(\left|\mathrm{H}_{1}-\mathrm{H}_{1}^{\prime}\right|>\sqrt{\delta}\right) \leq C \sqrt{\delta} .
$$


Now let $\rho$ denote the modulus of continuity of $\xi$. By (78) we have

$$
\left|\bar{\theta}_{n}^{\varepsilon}\left(\pi_{n} ; t, x\right)-\bar{\theta}_{n}^{\varepsilon}\left(\pi_{n}^{\prime} ; t^{\prime}, x^{\prime}\right)\right| \leq C \sup _{b \in \mathbb{L}_{L}^{0}(\mathbb{F})}\left[I_{1}(b)+I_{2}(b)+I_{3}\right],
$$

where

$$
\begin{aligned}
I_{1}(b) & :=\overline{\mathcal{E}}^{L}\left[\left|e^{\int_{t}^{T} \bar{b}_{r} d r}-e^{\int_{t^{\prime}}^{T} \bar{b}_{r}^{\prime} d r}\right|\right] \\
I_{2}(b) & :=\overline{\mathcal{E}}^{L}\left[\left|\int_{t}^{T} e^{\int_{s}^{T} \bar{b}_{r} d r} d s-\int_{t^{\prime}}^{T} e^{\int_{s}^{T} \bar{b}_{r}^{\prime} d r} d s\right|\right] ; \\
I_{3} & :=\overline{\mathcal{E}}^{L}\left[\rho\left(\left\|B^{\varepsilon}\left(\pi_{n}, t, x\right)-B^{\prime \varepsilon}\left(\pi_{n}^{\prime}, t^{\prime}, x^{\prime}\right)\right\|_{T}\right)\right] .
\end{aligned}
$$

Recall (80). Note that, by (79) and (82),

$$
\begin{aligned}
I_{1}(b) \leq & C \overline{\mathcal{E}}^{L}\left[\left|\int_{t}^{T} \bar{b}_{r} d r-\int_{t^{\prime}}^{T} \bar{b}_{r}^{\prime} d r\right|\right] \\
& =C \overline{\mathcal{E}}^{L}\left[\left|\int_{0}^{\tilde{\mathrm{H}}_{1}} \bar{b}_{t+r} d r-\int_{0}^{\tilde{\mathrm{H}}_{1}^{\prime}} \bar{b}_{t^{\prime}+r}^{\prime} d r+\int_{0}^{\tilde{T}} \bar{b}_{\mathrm{H}_{1}+r} d r-\int_{0}^{\tilde{T}^{\prime}} \bar{b}_{\mathrm{H}_{1}^{\prime}+r}^{\prime} d r\right|\right] \\
& \leq C \overline{\mathcal{E}}^{L}\left[\left|\tilde{\mathrm{H}}_{1}-\tilde{\mathrm{H}}_{1}^{\prime}\right|+\left|\tilde{T}-\tilde{T}^{\prime}\right|\right] \leq C \overline{\mathcal{E}}^{L}\left[\left|t-t^{\prime}\right|+\left|\mathrm{H}_{1}-\mathrm{H}_{1}^{\prime}\right|\right] \leq C \delta \\
I_{2}(b)= & \overline{\mathcal{E}}^{L}\left[\left|\int_{0}^{\tilde{\mathrm{H}}_{1}} e^{\int_{t+s}^{T} \bar{b}_{r} d r} d s-\int_{0}^{\tilde{\mathrm{H}}_{1}^{\prime}} e^{\int_{t^{\prime}+s}^{T} \bar{b}_{r}^{\prime} d r} d s+\int_{0}^{\tilde{T}^{2}} e^{\int_{\mathrm{H}_{1}+s}^{T} \bar{b}_{r} d r} d s-\int_{0}^{\tilde{T}^{\prime}} e^{\int_{\mathrm{H}_{1}^{\prime}+s}^{T} \bar{b}_{r}^{\prime} d r} d s\right|\right] \\
\leq & C \overline{\mathcal{E}}^{L}\left[\left|\int_{0}^{\tilde{\mathrm{H}}_{1} \wedge \tilde{\mathrm{H}}_{1}^{\prime}}\left[e^{\int_{t+s}^{T} \bar{b}_{r} d r}-e^{\int_{t^{\prime}+s} \bar{b}_{r}^{\prime} d r}\right] d s+\right| \tilde{\mathrm{H}}_{1}-\tilde{\mathrm{H}}_{1}^{\prime} \mid\right] \\
& \left.+\int_{0}^{\tilde{T} \wedge \tilde{T}^{\prime}}\left[e^{\int_{\mathrm{H}_{1}+s}^{T} \bar{b}_{r} d r}-e^{\int_{\mathrm{H}_{1}^{\prime}+s}^{T} \bar{b}_{r}^{\prime} d r}\right] d s|+| \tilde{T}-\tilde{T}^{\prime} \mid\right] \\
\leq & C \delta+C \overline{\mathcal{E}}^{L} \\
& {\left[\int_{0}^{\tilde{\mathrm{H}}_{1} \wedge \tilde{\mathrm{H}}_{1}^{\prime} \mid}\left|\int_{t+s}^{T} \bar{b}_{r} d r-\int_{t^{\prime}+s}^{T} \bar{b}_{r}^{\prime} d r\right| d s+\int_{0}^{T}\left|\int_{\mathrm{H}_{1}+s}^{T} \bar{b}_{r} d r-\int_{\mathrm{H}_{1}^{\prime}+s}^{T} \bar{b}_{r}^{\prime} d r\right| d s\right] } \\
\leq & C \delta,
\end{aligned}
$$

where the last estimate follows similar arguments as for $I_{1}(b)$. Then

$$
\left|\bar{\theta}_{n}^{\varepsilon}\left(\pi_{n} ; t, x\right)-\bar{\theta}_{n}^{\varepsilon}\left(\pi_{n}^{\prime} ; t^{\prime}, x^{\prime}\right)\right| \leq C \delta+C I_{3} .
$$

The estimate for $I_{3}$ is more involved. We first consider the case that $t>t_{n}$. Denote

$$
\delta_{0}:=\min _{1 \leq i \leq n}\left[t_{i}-t_{i-1}\right] \wedge\left[t-t_{n}\right]>0,
$$

and let $\delta_{1} \leq \frac{1}{2} \delta_{0}$ which will be specified later. Consider $\delta \leq \delta_{1}$, then

$$
\min _{1 \leq i \leq n}\left[t_{i}^{\prime}-t_{i-1}^{\prime}\right] \wedge\left[t^{\prime}-t_{n}^{\prime}\right] \geq \delta_{1}
$$


For any $m \geq 1$, by Lemma 3.4 we see that

$\sup _{\mathbb{P} \in \mathcal{P}_{L}} \mathbb{P}\left(\Omega_{m}^{c}\right) \leq \frac{C_{\varepsilon}}{m}$,

where $\Omega_{m}:=\{N \leq m\} \cap\left\{N^{\prime} \leq m\right\} \cap\left\{\sup _{0 \leq s_{1}<s_{2} \leq 2 T} \frac{\left|B_{s_{1}, s_{2}}\right|}{\left(s_{2}-s_{1}\right)^{\frac{1}{3}}} \leq m\right\} \cap\left\{\|B\|_{2 T} \leq m\right\}$,

and the constant $C_{\varepsilon}$ is independent of $\left(\pi_{n}, t, x\right)$ and $\left(\pi_{n}^{\prime}, t^{\prime}, x^{\prime}\right)$. Moreover, by (20) we have

$$
\sup _{\mathbb{P} \in \mathcal{P}^{L}}\left[\mathbb{P}\left(\mathrm{H}_{i+1}-\mathrm{H}_{i}<\delta_{1}, \mathrm{H}_{i+1}<T\right)+\mathbb{P}\left(\mathrm{H}_{i+1}^{\prime}-\mathrm{H}_{i}^{\prime}<\delta_{1}, \mathrm{H}_{i+1}^{\prime}<T\right)\right] \leq C_{\varepsilon} \delta_{1}, \quad i \geq 1 .
$$

This implies

$$
\begin{aligned}
\sup _{\mathbb{P} \in \mathcal{P}^{L}} \mathbb{P} & \left(\left[\bigcup_{i=1}^{N-2}\left\{\mathrm{H}_{i+1}-\mathrm{H}_{i}<\delta_{1}\right\} \cap\{N \leq m\}\right] \bigcup\left[\bigcup_{i=1}^{N^{\prime}-2}\left\{\mathrm{H}_{i+1}^{\prime}-\mathrm{H}_{i}^{\prime}<\delta_{1}\right\} \cap\left\{N^{\prime} \leq m\right\}\right]\right) \\
& \leq C_{\varepsilon} m \delta_{1} .
\end{aligned}
$$

Thus, by (82) and assuming $\sqrt{\delta} \leq \delta_{1}$,

$$
\begin{aligned}
& \sup _{\mathbb{P} \in \mathcal{P}^{L}} \mathbb{P}\left(\Omega_{m, \delta_{1}}^{c}\right) \leq C_{\varepsilon}\left[\frac{1}{m}+m \delta_{1}\right], \text { where } \\
& \Omega_{m, \delta_{1}}:=\Omega_{m} \bigcap\left\{\left|\mathrm{H}_{1}-\mathrm{H}_{1}^{\prime}\right| \leq \sqrt{\delta}\right\} \bigcap\left(\bigcap_{i=1}^{N-2}\left\{\mathrm{H}_{i+1}-\mathrm{H}_{i} \geq \delta_{1}\right\}\right) \bigcap\left(\bigcap_{i=1}^{N^{\prime}-2}\left\{\mathrm{H}_{i+1}^{\prime}-\mathrm{H}_{i}^{\prime} \geq \delta_{1}\right\}\right) .
\end{aligned}
$$

Denote $\tau_{0}:=0, \tau_{i}:=t_{i}, 1 \leq i \leq n, \tau_{n+i}:=\mathrm{H}_{i}, 1 \leq i \leq m$, and define $\tau_{i}^{\prime}$ similarly. Note that $t-t_{n} \geq \delta_{1}, t^{\prime}-t_{n}^{\prime} \geq \delta_{1}$, and $\mathrm{H}_{i}-\mathrm{H}_{i}^{\prime}=\mathrm{H}_{1}-\mathrm{H}_{1}^{\prime}, 1 \leq i<N \wedge N^{\prime}$, thanks to the third line of (79). Then, assuming $m \geq \sum_{i=1}^{n}\left[\left|x_{i}\right|+\left|x_{i}^{\prime}\right|\right]+|x|+\left|x^{\prime}\right|+n$, on $\Omega_{m, \delta_{1}}$ it holds that

$$
\begin{gathered}
\inf _{1 \leq i<n+N}\left[\tau_{i}-\tau_{i-1}\right] \geq \delta_{1}, \inf _{1 \leq i<n+N^{\prime}}\left[\tau_{i}^{\prime}-\tau_{i-1}^{\prime}\right] \geq \delta_{1}, \quad \sup _{1 \leq i<n+N \wedge N^{\prime}}\left|\tau_{i}-\tau_{i}^{\prime}\right| \leq \sqrt{\delta} ; \\
\sup _{1 \leq i \leq N}\left|X_{i}\right| \leq 2 m, \quad \sup _{1 \leq i \leq N^{\prime}}\left|X_{i}^{\prime}\right| \leq 2 m, \quad \sup _{1 \leq i<n+N \wedge N^{\prime}}\left|X_{i}-X_{i}^{\prime}\right| \leq C m \delta^{\frac{1}{6}} .
\end{gathered}
$$

We now estimate $I_{s}^{3}:=\left|B_{s}^{\varepsilon}\left(\pi_{n}, t, x\right)-B_{s}^{\varepsilon}\left(\pi_{n}^{\prime}, t^{\prime}, x^{\prime}\right)\right|$ on the set $\Omega_{m, \delta_{1}}$. Without loss of generality we assume $\sqrt{\delta} \leq \frac{\delta_{1}}{2}, \mathrm{H}_{1}^{\prime} \leq \mathrm{H}_{1}$ and thus $N \leq N^{\prime}$. We estimate $I_{s}^{3}$ in several cases. 
Case 1. $s \in\left[\tau_{i}+\sqrt{\delta}, \tau_{i+1}-\sqrt{\delta}\right]$ for some $0 \leq i \leq n+N-2$. Then, noting that $\left|\tau_{j}-\tau_{j}^{\prime}\right| \leq \sqrt{\delta}$ for $j=i, i+1$, we have $s \in\left[\tau_{i}^{\prime}, \tau_{i+1}^{\prime}\right]$ and thus

$$
\begin{aligned}
I_{s}^{3}= & \left|X_{i}+\frac{s-\tau_{i}}{\tau_{i+1}-\tau_{i}}\left[X_{i+1}-X_{i}\right]-X_{i}^{\prime}-\frac{s-\tau_{i}^{\prime}}{\tau_{i+1}^{\prime}-\tau_{i}^{\prime}}\left[X_{i+1}^{\prime}-X_{i}^{\prime}\right]\right| \\
= & \mid\left[X_{i}-X_{i}^{\prime}\right]+\frac{s-\tau_{i}^{\prime}}{\tau_{i+1}^{\prime}-\tau_{i}^{\prime}}\left[\left(X_{i+1}-X_{i}\right)-\left(X_{i+1}^{\prime}-X_{i}^{\prime}\right)\right] \\
& +\left[\left[\frac{s-\tau_{i}}{\tau_{i+1}-\tau_{i}}-\frac{s-\tau_{i}^{\prime}}{\tau_{i+1}-\tau_{i}}\right]+\left[\frac{s-\tau_{i}^{\prime}}{\tau_{i+1}-\tau_{i}}-\frac{s-\tau_{i}^{\prime}}{\tau_{i+1}^{\prime}-\tau_{i}^{\prime}}\right]\right]\left[X_{i+1}-X_{i}\right] \mid .
\end{aligned}
$$

Now by (90) we can easily see that $I_{s}^{3} \leq C m \delta^{\frac{1}{6}}+\frac{C m}{\delta_{1}} \sqrt{\delta}$.

Case 2. $\left|s-\tau_{i}\right| \leq \sqrt{\delta}$ for some $0 \leq i<n+N$. Then $\left|s-\tau_{i}^{\prime}\right| \leq 2 \sqrt{\delta}$, and

$$
I_{s}^{3} \leq\left|B_{s}^{\varepsilon}\left(\pi_{n}, t, x\right)-B_{\tau_{i}}^{\varepsilon}\left(\pi_{n}, t, x\right)\right|+\left|X_{i}-X_{i}^{\prime}\right|+\left|B_{\tau_{i}^{\prime}}^{\varepsilon}\left(\pi_{n}^{\prime}, t^{\prime}, x^{\prime}\right)-B_{s}^{\varepsilon}\left(\pi_{n}^{\prime}, t^{\prime}, x^{\prime}\right)\right| .
$$

Case 2.1. Assume $0 \leq i \leq n$. Then (92) and (90) lead to

$$
I_{s}^{3} \leq \frac{C m}{\delta_{1}}\left[\left|s-\tau_{i}\right|+\left|s-\tau_{i}^{\prime}\right|\right]+C m \delta^{\frac{1}{6}} \leq C m \delta^{\frac{1}{6}}+\frac{C m}{\delta_{1}} \sqrt{\delta}
$$

Case 2.2. Assume $n<i<n+N$. Note that, when $s>\tau_{i}$,

$$
\left|B_{\tau_{i}, s}^{\varepsilon}\left(\pi_{n}, t, x\right)\right|=\frac{s-\tau_{i}}{\tau_{i+1}-\tau_{i}}\left|X_{i+1}-X_{i}\right| \leq \frac{s-\tau_{i}}{\tau_{i+1}-\tau_{i}} m\left(\tau_{i+1}-\tau_{i}\right)^{\frac{1}{3}} \leq m\left(s-\tau_{i}\right)^{\frac{1}{3}} \leq m \delta^{\frac{1}{6}} .
$$

Similarly we have the other related estimates. Then (92) and (90) lead to $I_{s}^{3} \leq C m \delta^{\frac{1}{6}}$.

Case 3. $s \in\left[\tau_{n+N-1}+\sqrt{\delta}, T\right]$ and $T-\tau_{n+N-1} \leq \delta^{\frac{1}{4}}$. Then $s-\tau_{n+N-1} \leq \delta^{\frac{1}{4}}$ and $0 \leq s-\tau_{n+N-1}^{\prime} \leq \delta^{\frac{1}{4}}+\sqrt{\delta} \leq 2 \delta^{\frac{1}{4}}$. When $N^{\prime}=N$, then of course $s \in$ $\left[\tau_{n+N-1}^{\prime}, \tau_{n+N}^{\prime}\right]$. When $\bar{N}<N^{\prime}$, then $\tau_{n+N}^{\prime}-\tau_{n+N^{\prime}-1}^{\prime} \geq \delta_{1}$ and thus we still have $s \in\left[\tau_{n+N-1}^{\prime}, \tau_{n+N}^{\prime}\right]$ whenever $2 \delta^{\frac{1}{4}} \leq \delta_{1}$. Following the arguments in Case 2.2, by (92) and (90) we can easily see that $I_{s}^{3} \leq C m \delta^{\frac{1}{12}}$.

Case 4. $s \in\left[\tau_{n+N-1}+\sqrt{\delta}, T\right]$ and $T-\tau_{n+N-1} \geq \delta^{\frac{1}{4}}$. Denote $i:=n+N-1$ for notational simplicity, then $\tau_{i+1}=T$. Similar to (79), by (76) one can easily see that $\tau_{i+1}^{\prime}-\tau_{i}^{\prime} \geq \tau_{i+1}-\tau_{i}$. Then, together with (90) we have

$$
\begin{aligned}
\tau_{i+1}^{\prime}-\tau_{i}^{\prime} & \geq T-\tau_{i} \geq \delta^{\frac{1}{4}}, \quad \tau_{i+1}-\tau_{i+1}^{\prime} \\
& \leq \tau_{i}-\tau_{i}^{\prime} \leq \sqrt{\delta}, \quad \tau_{i+1}^{\prime}-\tau_{i}^{\prime} \leq T-\tau_{i}+\sqrt{\delta}
\end{aligned}
$$

Case 4.1. Assume $s \in\left[\tau_{i}^{\prime}, \tau_{i+1}^{\prime}\right]$. Then, by (91), (90), and the first two inequalities of (93), we have $I_{s}^{3} \leq C m \delta^{\frac{1}{6}}+\frac{C m}{\delta^{\frac{1}{4}}} \sqrt{\delta} \leq C m \delta^{\frac{1}{4}}$.

Case 4.2. Assume $s \notin\left[\tau_{i}^{\prime}, \tau_{i+1}^{\prime}\right]$. In this case, we must have $N^{\prime}>N$ and thus $\tau_{i+1}^{\prime}-\tau_{i}^{\prime} \geq \delta_{1}$. By the second inequality of (93) we see that $T-\tau_{i+1}^{\prime} \leq \sqrt{\delta}<\delta_{1}$, 
then we must have $N^{\prime}=N+1$ and thus $s \in\left[\tau_{i+1}^{\prime}, \tau_{i+2}^{\prime}\right]$. By (90) and (93) again we can see that

$$
T-\tau_{i} \geq \delta_{1}-\sqrt{\delta}, \quad 0 \leq s-\tau_{i+1}^{\prime} \leq T-\tau_{i+1}^{\prime} \leq \sqrt{\delta}, \quad T-s \leq T-\tau_{i+1}^{\prime} \leq \sqrt{\delta},
$$

Then, recalling the notations in (81),

$$
\begin{aligned}
I_{s}^{3} & =\left|X_{i}+B_{\tilde{T}+\tau_{i}, \tilde{T}+T}-\frac{T-s}{T-\tau_{i}} B_{\tilde{T}+\tau_{i}, \tilde{T}+T}-X_{i}^{\prime}-B_{\tilde{T}^{\prime}+\tau_{i}^{\prime}, \tilde{T}^{\prime}+\tau_{i+1}^{\prime}}-\frac{s-\tau_{i+1}^{\prime}}{T-\tau_{i+1}^{\prime}} B_{\tilde{T}^{\prime}+\tau_{i+1}^{\prime}, \tilde{T}^{\prime}+T}\right| \\
& \leq C m \delta^{\frac{1}{6}}+\frac{T-s}{T-\tau_{i}}\left|B_{\tilde{T}+\tau_{i}, \tilde{T}+T}\right|+\left|B_{\tilde{T}+\tau_{i}, \tilde{T}+T}-B_{\tilde{T}^{\prime}+\tau_{i}^{\prime}, \tilde{T}^{\prime}+\tau_{i+1}^{\prime}}\right|+C m\left|T-\tau_{i+1}^{\prime}\right|^{\frac{1}{3}} \\
& \leq C m \delta^{\frac{1}{6}}+C m \frac{\sqrt{\delta}}{\delta_{1}-\sqrt{\delta}}+C\left[\left|\tilde{T}-\tilde{T}^{\prime}\right|^{\frac{1}{3}}+\left|\tau_{i}-\tau_{i}^{\prime}\right|^{\frac{1}{3}}+\left|T-\tau_{i+1}^{\prime}\right|^{\frac{1}{3}}\right] \leq C m \delta^{\frac{1}{6}}+C m \frac{\sqrt{\delta}}{\delta_{1}} .
\end{aligned}
$$

Combining all the cases together, we have

$$
I_{s}^{3} \leq C m \delta^{\frac{1}{12}}+C m \frac{\sqrt{\delta}}{\delta_{1}} \quad \text { on } \Omega_{m, \delta_{1}} \cap\left\{\mathrm{H}_{1}^{\prime} \leq \mathrm{H}_{1}\right\} .
$$

We may get the same estimate on $\Omega_{m, \delta_{1}} \cap\left\{\mathrm{H}_{1} \leq \mathrm{H}_{1}^{\prime}\right\}$. Plug this and (89) into (85), we obtain

$$
\left|\bar{\theta}_{n}^{\varepsilon}\left(\pi_{n} ; t, x\right)-\bar{\theta}_{n}^{\varepsilon}\left(\pi_{n}^{\prime} ; t^{\prime}, x^{\prime}\right)\right| \leq C \delta+\frac{C_{\varepsilon}}{m}+C_{\varepsilon} m \delta_{1}+C m \delta \frac{1}{12}+C m \frac{\sqrt{\delta}}{\delta_{1}} .
$$

Set $\delta_{1}:=\frac{1}{m^{2}} \wedge \frac{\delta_{0}}{2}$. Then, whenever $\delta \leq \frac{1}{m^{48}}$,

$$
\left|\bar{\theta}_{n}^{\varepsilon}\left(\pi_{n} ; t, x\right)-\bar{\theta}_{n}^{\varepsilon}\left(\pi_{n}^{\prime} ; t^{\prime}, x^{\prime}\right)\right| \leq \frac{C_{\varepsilon}}{m}+C_{\varepsilon} m^{3} \delta^{\frac{1}{12}} \leq \frac{C_{\varepsilon}}{m} .
$$

Since $m \geq 1$ is arbitrary, we see that $\bar{\theta}_{n}^{\varepsilon}$ is continuous at $\left(\pi_{n}, t, x\right)$, in the case that $t>t_{n}$.

In the case $t=t_{n}$ and thus $x=0$, we modify (86) as

$$
\delta_{0}:=\min _{1 \leq i \leq n}\left[t_{i}-t_{i-1}\right]>0 .
$$

Note that, in this case the inequality $\left|x^{\prime}\right| \leq \delta$ holds and one can easily see that (88) still holds true for $i=0$. Following almost the same arguments as in previous case we may prove that $\bar{\theta}_{n}^{\varepsilon}$ is continuous at $\left(\pi_{n}, t_{n}, 0\right)$.

Acknowledgements Research supported in part by NSF grant DMS 1413717.

\section{Authors' contributions}

Both authors read and approved the final manuscript.

\section{Competing interests}

The authors declare that they have no competing interests. 


\section{References}

Barles, G, Buckdahn, R, Pardoux, E: Backward stochastic differential equations and integral-partial differential equations. Stoch. Stoch. Rep 60, 57-83 (1997)

Bayraktar, E, Yao, S: Optimal Stopping with Random Maturity under Nonlinear Expectations. preprint (2016). arXiv:1505.07533

Cont, R, Fournie, D: Functional Itô calculus and stochastic integral representation of martingales. Ann. Probab 41, 109-133 (2013)

Cosso, A, Russo, F: Strong-viscosity solutions: Semilinear parabolic PDEs and path-dependent PDEs. preprint (2016). arXiv:1505.02927

Crandall, MG, Ishii, H, Lions, P-L: User's guide to viscosity solutions of second order partial differential equations. Bull. Amer. Math. Soc. (NS) 27, 1-67 (1992)

Dupire, B: Functional Itô calculus (2009). papers.ssrn.com

Ekren, I, Keller, C, Touzi, N, Zhang, J: On Viscosity Solutions of Path Dependent PDEs. Ann. Probab 42, 204-236 (2014a)

Ekren, I, Touzi, N, Zhang, J: Optimal Stopping under Nonlinear Expectation. Stochastic Process. Appl 124, 3277-3311 (2014b)

Ekren, I, Touzi, N, Zhang, J: Viscosity Solutions of Fully Nonlinear Parabolic Path Dependent PDEs: Part I. Ann. Probab 44, 1212-1253 (2016a)

Ekren, I, Touzi, N, Zhang, J: Viscosity Solutions of Fully Nonlinear Parabolic Path Dependent PDEs: Part II. Ann. Probab 44, 2507-2553 (2016b)

Fleming, W, Soner, HM Controlled Markov Processes and Viscosity Solutions, 2nd ed. Springer, New York (2006)

Fleming, W, Souganidis, PE: On The Existence of Value Functions of Two-Player, Zero-Sum Stochastic Differential Games. Indiana Univ. Math. J 38, 293-314 (1989)

Mikulevicious, R: On the convergence of diffusions, Stochastic Differential Systems, pp. 176-186. Springer-Verlag, Berlin (1987)

Mikulevicius, R, Rozovskii, B: Martingale problems for stochastic PDE's. Stochastic partial differential equations: six perspectives, pp. 243-325 (1999). Math. Surveys Monogr., 64, Amer. Math. Soc., Providence, RI

Peng, S: Stochastic Hamilton-Jacobi-Bellman Equations. SIAM J. Control Optim 30, 284-304 (1992)

Peng, S: Open problems on backward stochastic differential equations. Control of distributed parameter and stochastic systems, pp. 265-273. Springer, US (1999)

Peng, S: Backward stochastic differential equation, nonlinear expectation and their applications. Proceedings of the International Congress of Mathematicians, Hyderabad, India (2010)

Peng, S: Note on Viscosity Solution of Path-Dependent PDE and G-Martingales. preprint, arXiv:1106.1144 (2011)

Peng, S, Song, Y: G-Expectation Weighted Sobolev Spaces, Backward SDE and Path Dependent PDE. J. Math. Soc. Japan 67, 1725-1757 (2015)

Peng, S, Wang, F: BSDE, Path-dependent PDE and Nonlinear Feynman-Kac Formula. Sci. China Math 59, 19-36 (2016)

Pham, T, Zhang, J: Some Norm Estimates for Semimartingales. Electron. J. Probab 18, 1-25 (2013)

Pham, T, Zhang, J: Two Person Zero-sum Game in Weak Formulation and Path Dependent Bellman-Isaacs Equation. SIAM J. Control Optim 52, 2090-2121 (2014)

Qiu, J: Weak Solution for a Class of Fully Nonlinear Stochastic Hamilton-Jacobi-Bellman Equations. preprint (2016). arXiv:1410.6967

Ren, Z: Perron's method for viscosity solutions of semilinear path dependent PDEs, Stochastics: An International Journal of Probability and Stochastic Processes (2016)

Ren, Z, Touzi, N, Zhang, J: An Overview of Viscosity Solutions of Path-Dependent PDEs. Stochastic Anal. Appl 100, 397-453 (2014)

Ren, Z, Touzi, N, Zhang, J: Comparison of Viscosity Solutions of Semilinear Path-Dependent PDEs, preprint (2016a). arXiv:1410.7281

Ren, Z, Touzi, N, Zhang, J: Comparison of Viscosity Solutions of Fully Nonlinear Degenerate Parabolic Path-dependent PDEs, preprint (2016b). arXiv:1511.05910 